\title{
The Effectiveness of Dog Population Management: A Systematic Review
}

\author{
Lauren M. Smith ${ }^{1}{ }^{1}$, Sabine Hartmann ${ }^{2}$, Alexandru M. Munteanu ${ }^{2}$, Paolo Dalla Villa ${ }^{3}$, \\ Rupert J. Quinnell ${ }^{1}$ and Lisa M. Collins ${ }^{1, *}$ \\ 1 Faculty of Biological Sciences, University of Leeds, Leeds LS2 9JT, UK; bslsmi@leeds.ac.uk (L.M.S.); \\ R.J.Quinnell@leeds.ac.uk (R.J.Q.) \\ 2 VIER PFOTEN International, 1150 Vienna, Austria; Sabine.Hartmann@vier-pfoten.org (S.H.); \\ alex.munteanu@vier-pfoten.org (A.M.M.) \\ 3 Istituto Zooprofilattico Sperimentale dell'Abruzzo e del Molise “G. Caporale”, 64100 Teramo, Italy; \\ p.dallavilla@izs.it \\ * Correspondence: L.Collins@leeds.ac.uk
}

Received: 4 November 2019; Accepted: 18 November 2019; Published: 22 November 2019

Simple Summary: Domestic dogs are abundant worldwide-as owned pets, unowned strays, and feral animals. High numbers of free-roaming dogs can be a concern because of the risks they pose to public health, animal welfare, and wildlife. Using a systematic review process, we investigated what the research published to date can tell us about the effectiveness of different dog population management methods. We found that management methods for dog populations have been researched in multiple countries worldwide, using a wide range of indicators to assess method effectiveness. We outline the results and suggest improvements to help guide future dog population management policy.

\begin{abstract}
The worldwide population of domestic dogs is estimated at approximately 700 million, with around $75 \%$ classified as "free-roaming". Where free-roaming dogs exist in high densities, there are significant implications for public health, animal welfare, and wildlife. Approaches to manage dog populations include culling, fertility control, and sheltering. Understanding the effectiveness of each of these interventions is important in guiding future dog population management. We present the results of a systematic review of published studies investigating dog population management, to assess: (1) where and when studies were carried out; (2) what population management methods were used; and (3) what was the effect of the method. We evaluated the reporting quality of the published studies for strength of evidence assessment. The systematic review resulted in a corpus of 39 papers from 15 countries, reporting a wide disparity of approaches and measures of effect. We synthesised the management methods and reported effects. Fertility control was most investigated and had the greatest reported effect on dog population size. Reporting quality was low for power calculations $(11 \%)$, sample size calculations $(11 \%)$, and the use of control populations $(17 \%)$. We provide recommendations for future studies to use common metrics and improve reporting quality, study design, and modelling approaches in order to allow better assessment of the true impact of dog population management.
\end{abstract}

Keywords: Canis familiaris; free-roaming; stray; population control; catch-neuter-release; sheltering; culling 


\section{Introduction}

\subsection{The Dog Population}

Dogs (Canis familiaris) have a global distribution and an estimated total population size of around 700 million [1]. Dogs have one of two ownership states-either they are owned or they are unowned. The owned population is dependent upon humans for food, water, and shelter and may have one or more owner (e.g., "community dogs"). The owned dog population includes both dogs that are restricted in their movement to a limited area (e.g., within a fenced yard or under human supervision on walks), and those that are free to roam unrestricted, without human supervision [2]. Unowned dogs (often referred to as stray) do not have an owner but may still depend upon humans directly or indirectly for resources such as food, water, and shelter [2]. Similar to owned dogs, the unowned population's movements may be restricted or unrestricted-dogs housed in shelters have restricted movement, but street-dwelling dogs have unrestricted movement. Unrestricted dogs are particularly important as the lack of restriction allows them to roam freely, to mate, and to reproduce. This group is commonly referred to as the free-roaming dog population and includes unrestricted owned and unrestricted unowned dogs [3].

Approximately $75 \%$ of dogs across the world fit into this free-roaming dog category [1]. Free-roaming dog abundance varies greatly between countries, relating to the habitat type (urban/rural) and human population (e.g., density and cultural/social factors) [2]. Although dog densities vary globally, they can exist in high numbers. For example, densities as high as 719 dogs $\mathrm{km}^{-2}$ have been estimated in Maharashtra in India [4]. Where free-roaming dogs exist in high densities, they can be considered an issue in terms of public health (e.g., transmission of rabies and other zoonotic pathogens) [5-7], the environment (e.g., threatening the survival of wildlife) [8-10], and the animals' welfare state [2,11-13]. Population management therefore typically focusses on free-roaming dogs [14] to control the population size and-depending on the approach taken-to improve dog health and welfare and mitigate against public health and environmental problems $[15,16]$.

\subsection{Reasons for Managing Free-Roaming Dog Populations}

\subsubsection{The Impact of Free-Roaming Dogs on Public Health}

Free-roaming dogs are associated with the transmission of a number of zoonotic pathogens [17-21], dog bite injuries [22-24], and road traffic accidents [2,25]. Dogs are responsible for transmitting over 300 zoonoses to humans $[26,27]$. They are perhaps best known for the role they play in the spread and maintenance of the rabies virus [28]. This virus is responsible for an estimated 60,000 human deaths per year amounting to an annual economic cost of 8.6 billion US dollars [29]. Dogs are a primary reservoir host of this virus and account for $99 \%$ of human-rabies transmissions [28]. Other notable zoonoses include Leishmania infantum, the causative agent of zoonotic visceral leishmaniasis [30]; Echinococcus spp. (E. granulosus and E. multilocularis) [31], which causes echinococcosis; and Toxocara canis, which causes toxocariasis [32].

\subsubsection{The Impact of Free-Roaming Dogs on Wild and Domestic Animals}

Free-roaming dogs can compromise the conservation of wild animals through a combination of pathogen pollution (i.e., the spread of pathogens to naive hosts) [8,33,34], predation [35], competition [36], and hybridisation [9,37,38]. It is estimated that dogs have played a role in the extinction of 11 vertebrate species and threaten the survival of at least 188 more species [10]. In addition to the negative consequences that canine pathogens have on human health, these pathogens can have an important impact on the conservation of endangered species. For example, rabies spread by domestic dogs can threaten Ethiopian wolf (Canis simensis) [8] and African wild dog (Lycaon pictus) [33] populations. The taxonomic relatedness of the domestic dog to other canids, such as dingoes (Canis lupus dingo) and wolves (Canis lupus ssp.), is also of concern when dogs are free-roaming. Inter-species 
breeding can result in hybridisation and threaten species survival [9,37,39]. In addition, dogs have been responsible for the reduction of other species through predation and competition $[1,40,41]$. For example, predation by dogs resulted in the decrease of mountain gazelle (Gazella gazella gazella) in Israel [42], puda (Puda puda) in Chile [43], and kiwi (Apteryx australis mantelli) in New Zealand [44].

Domestic dogs can also be responsible for the killing of livestock [45-49]; in particular, small-and medium-bodied livestock such as sheep, goats, and donkeys [45-49]. The loss of livestock contributes to substantial economic losses [45]. For example, in the USA, this amounts to over $\$ 620$ million US dollars annually [48]. The financial consequences can be particularly problematic in areas of low-income communities [45]. Additionally, the loss of livestock can increase human-wildlife conflict, as predation by dogs is often mistaken for that of other species, such as wolves [45,46,49] or snow leopards [45]. This can hinder local support for conservation programs.

\subsubsection{Health and Welfare of Free-Roaming Dogs}

Free-roaming dogs can often experience poor health and welfare conditions. In particular, unowned free-roaming dogs may have an inadequate diet and a high prevalence of starvation and dehydration [2,13]. For example, in India, around $49 \%$ of free-roaming dogs have an emaciated body condition [50]. Unowned free-roaming dogs can still be dependent on humans for resources, either directly through feeding or indirectly through the provision of food in human waste [51]. The high prevalence of emaciated body condition state in free-roaming dogs that occurs in some areas may be due to poor quantity or quality of food resources and a high disease burden [50,52]. Free-roaming dogs, particularly those that are unowned, lack veterinary care (such as vaccination or antiparasitics) and are therefore more susceptible to disease. High prevalence of skin conditions and ectoparasites have been reported in several populations $[50,53]$. Canine transmissible venereal tumour disease is also a welfare concern in free-roaming dog populations, with the prevalence estimated at around $1 \%$ in dog populations in Africa, Asia, South America, and Central America [54], although the prevalence has been estimated to be as high as $15 \%$ in female dogs in some free-roaming populations [55]. Additional health and welfare risks to free-roaming dogs include injury caused by road traffic accidents, abusive treatment by locals [3], and inhumane methods of removal (e.g., poisoning, electrocution, drowning, or carbon monoxide poisoning [56]).

\subsection{Methods of Managing Dog Populations}

\subsubsection{Responsible Groups and Motivations for Dog Population Management}

Different groups (e.g., researchers, animal welfare organisations, or government agencies) are often responsible for setting up dog population management programs [15]. They manage the population in three main ways: culling, long-term sheltering, and fertility control of free-roaming dogs [14,15]. In addition, programs may include a focus on public education of responsible ownership and taxation of dog ownership. Different countries, as well as different regions within a country, may vary in their objectives for carrying out dog population management programs [14,15], such as: reducing the number of free-roaming dogs; increasing awareness of responsible ownership practices; or improving the health of the free-roaming dog population. These objectives may be underpinned by: dog-centric motives, such as improving dog health and welfare; human-centric motives, such as the control of zoonotic disease [14] and reduced prevalence of dog bite injuries; and wildlife-centric motives, such as reducing the risk to the conservation of other species.

\subsubsection{Culling}

Historically, culling has been the primary method used to reduce numbers of free-roaming dogs [57]. Culling is the episodic removal and killing of individuals for the purpose of population reduction. The World Health Organisation published guidelines in 1990 discouraging the use of culling and recommending alternative methods (e.g., registration and identification, vaccination, public 
education, and sterilisation) [58]. Despite these recommendations, many countries continue to use culling as a primary method of population control [15]. Injectable barbiturates are more commonly used in high- and upper-middle-income countries [15], whereas poisoning and shooting are often used in lower-middle- and low-income countries [15]. National law in some countries (e.g., Bulgaria [59], Italy [60], and Kosovo [61])) prohibits the killing of dogs for the purpose of population control.

\subsubsection{Sheltering}

In some countries, sheltering free-roaming dogs is the most common method of dog population control. Similar to culling, sheltering aims to reduce the free-roaming dog population size by removal of dogs. Ultimately, sheltered dogs may be: (i) euthanised; (ii) adopted; or (iii) permanently stay in the shelter. Shelters are commonplace globally and may be government-run (public shelters), privately-run, or operated by NGOs. The numbers of dogs coming into the shelter are often greater than the number of dogs going out, for example to be rehomed [62-64]. This results in either lifelong stays in the shelter or euthanasia [62-64]. As national law in some countries prohibits euthanasia of healthy animals, this can lead to long-term sheltering and overcrowding [65]. Moreover, the use of shelters to house dogs is costly and, as such, more commonly employed in high- and upper-middle-income countries [15]. Due to the expense, this method may be unsuitable in lower-middle- and low-income countries [15].

\subsubsection{Fertility Control}

Fertility control can be achieved through surgical or chemical sterilisation or contraception [66]. Surgical sterilisation through the catch-neuter-release (CNR) of free-roaming dogs is the predominant method of fertility control. This method involves collecting free-roaming dogs and carrying out spay or castration surgery in either a fixed-location or mobile clinic. CNR has been carried out in several countries and states, for example in Italy [67], India [68-70], Bangladesh [71], Sri Lanka [72], and Brazil [73]. Surgical sterilisation is generally more socially acceptable than culling. However, in some locations, there can be conflict between locals and the groups/agencies conducting CNR, as some owned free-roaming dogs are caught and neutered against their owner's wishes [74]. In some communities, owners are against the surgical sterilisation of dogs due to their religious beliefs or the misunderstanding that neutering causes undesired behavioural changes $[75,76]$. In addition, CNR has associated expense, as it requires skilled staff, clinical facilities, and medicines.

\subsection{Study Aims}

We conducted a systematic review to synthesise the existing evidence of the effectiveness of different dog population management methods. In this review, we describe: (1) where and when the impact of dog population management has been assessed; (2) what management methods have been used; and (3) what effect the management method had on: (i) the dog population size; (ii) dog health and welfare; (iii) public health risk; (iv) public attitude; and (v) risk to wildlife populations. The effectiveness of dog population management depends upon the management intensity (coverage and length of management); therefore, we reported the effects in relation to these criteria wherever possible. In addition, we evaluated the reporting quality of the relevant published studies to allow weighting of evidence for future decision-making.

\section{Materials and Methods}

\subsection{Search Strategy}

We conducted an initial literature search in February 2017, using the following search engines: Web of Science; ProQuest (Applied Social Sciences Index \& Abstracts, PAIS Index, Sociological Abstracts, and Worldwide Political Science Abstracts); LILACS; and Google Scholar (results from Google Scholar were limited to the first 50 pages, due to the high volume of returned literature and lack of relevancy). The search used key words relating to dog population management (Table S1). We carried out a 
second search using the same search engines, keywords, and eligibility requirements in January 2019 to include any papers published in the interim period.

\subsection{Eligibility Requirements}

A single corpus of all returned literature was compiled across the searches and cleaned of any duplications prior to filtering. Entries were filtered in three stages, based on the relevance to the study aims. These stages involved assessing the paper's: (1) title; (2) abstract; and (3) full text. At each stage, papers were included or excluded depending on their match to the following inclusion criteria: (i) one of the primary aims of the literature was to assess, describe, investigate, or compare the impact of unowned free-roaming dog population management, in terms of dog population demographics, dog health and welfare, public attitude, or public health risk; (ii) the study design was observational, intervention or modelling; and (iii) was primary literature. Papers were excluded from the review if: (i) they were not a primary research source; (ii) their study design was systematic review, meta-analysis, lab intervention, or case report; or (iii) they assessed, described, or compared only owned dogs that were not free-roaming (i.e., restricted, owned dogs). This was assessed at Stage (1) (title stage) depending on whether the title included the key words (Table S1) indicating that the paper met the inclusion criteria. At Stages (2) (abstract) and (3) (full text), this was assessed by whether the text met the above-stated inclusion and exclusion criteria. Studies in all languages were considered, although searches were conducted with keywords in English only. There was no restriction on date of publication.

Papers that passed through all three filtering stages were included for review and are referred to as the final corpus. At Stages (1) and (2) of the filtering process, a second reviewer assessed 3\% of the papers (Stage (1) $=150$ of 4629 papers and Stage (2) $=30$ of 923 papers) to check the level of inter-rater inclusion/exclusion agreement. Any papers that were disagreed upon were disputed and a decision reached jointly by both reviewers (details in the Supplementary Materials).

To increase the possibility of capturing all relevant papers, references from papers in the final corpus were screened using the above three-stage filtering process. All references that matched the above inclusion/exclusion criteria were included in the final corpus.

\subsection{Information Extraction}

The following information was extracted from the final corpus: (i) year of publication, country of study, and its economic status (defined by The World Bank 2019 country income classification [77]); (ii) study impact category (dog health and welfare, dog demographics, public attitude, public health, or wildlife), and dog population management method (culling, sheltering, fertility control, or a combination of methods); and (iii) methods, measurements, and study reporting quality. Reporting quality was assessed based on guidelines from Preferred Reporting Items for Systematic Reviews and Meta-Analyses (PRISMA) [78] and Animals in Research: Reporting In Vivo Experiments (ARRIVE) [79]. For the final corpus, we assessed quality based on: study design, reporting of aims/hypotheses, appropriate study outcome (as defined by [16]), and definition of study population. Study populations were classified into: (i) unowned, free-roaming; (ii) owned, free-roaming; (iii) unowned, restricted; (iv) owned restricted; (v) undefined (i.e., the paper did not report which population was under investigation); or a combination of the five categories.

\subsection{Evaluating Study Design and Reporting Quality}

Where appropriate for the study design, we assessed the study and reporting quality based on: the presence/absence of a power calculation, presence/absence of a sample size calculation, inclusion of a control population, accounting for inter-observer reliability, and reporting of baseline characteristics. 


\section{Results}

\subsection{Year of Publishing, Country of Study and Economic Status}

The systematic review resulted in an initial (pre-filtered) corpus of 4863 papers, which we reduced following the three-stage filtering process to 36 papers (Figure 1). To ensure key papers were not missed, the references of included papers were reviewed using the same inclusion/exclusion criteria. This resulted in three additional papers and a final corpus of 39 papers. The final corpus comprised 36 peer-reviewed papers and three theses (two Masters of Science and one Masters of Veterinary Medicine). The papers were published between 1977 and 2018, with 82\% published between 2008 and 2018.

Most of the studies were carried out or used data from locations within a single country (87\%). These were located in 15 different countries across Africa (3\%), Asia (39\%), Central America (3\%), Europe (18\%), North America (10\%), and South America (15\%), in countries that were high income $(27 \%)$, upper-middle income (38\%), lower-middle income $(32 \%)$, and low income (3\%). A high proportion of the studies was conducted in India (26\%) (Table S2). Three studies used data from multiple countries ( $8 \%$ ) and two studies did not specify a country (5\%).

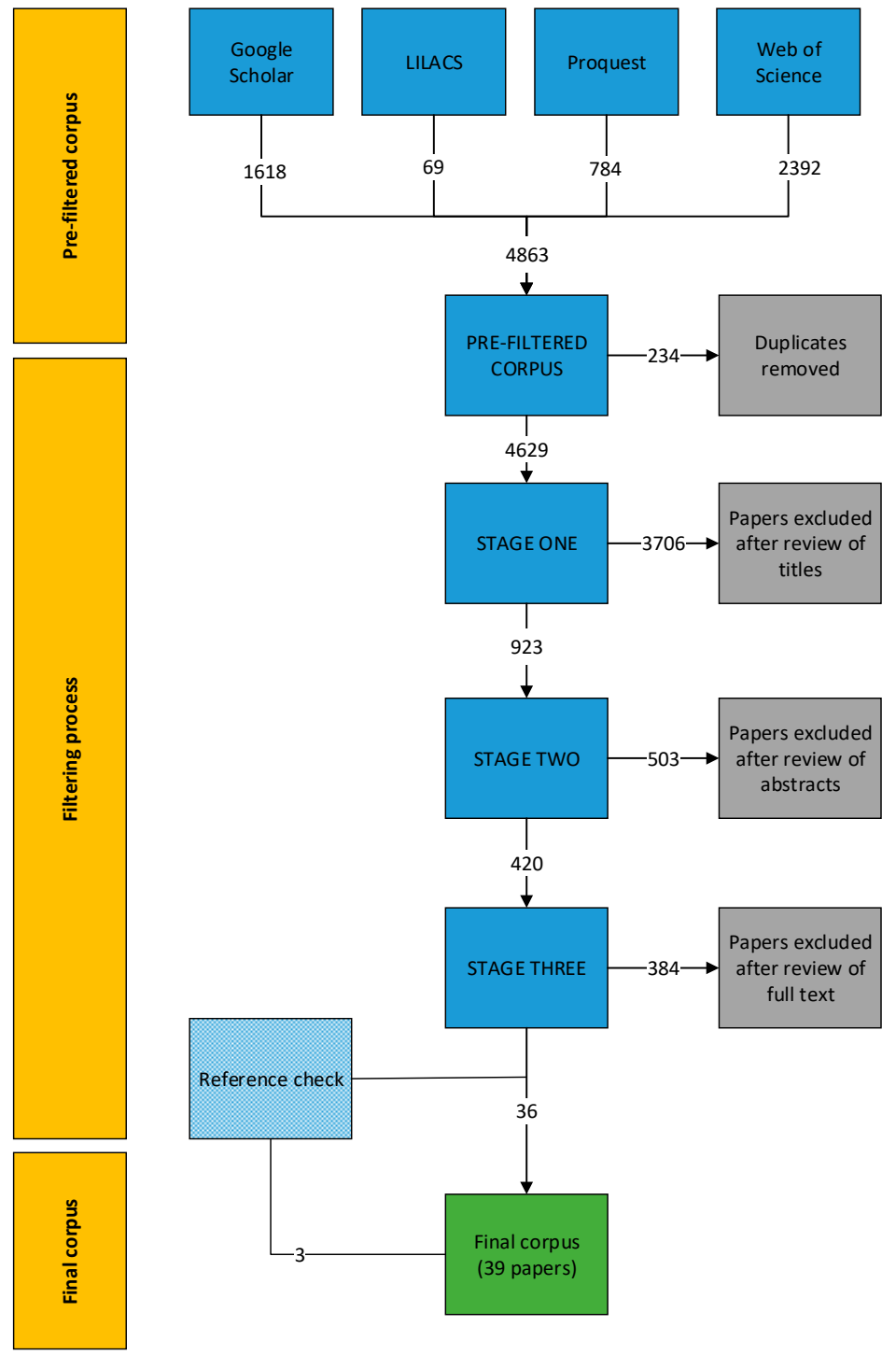

Figure 1. Number of papers included and excluded at each stage of the systematic review process. Grey boxes indicate the number excluded at each stage and the green box indicates the number of papers included in the final corpus. 


\subsection{Dog Population Management Methods and Impacts}

\subsubsection{Dog Population Management Methods}

The management methods studied in the final corpus included: fertility control through neutering and immunocontraceptives (13 papers, 33\%); culling (indiscriminate culling and culling of infected dogs: (7 papers, $18 \%)$ ); sheltering (2 papers, $5 \%$ ); and taxation (1 paper, $3 \%$ ) (Table 1 ). Combinations of methods were also studied: fertility control and sheltering ( 9 papers, $23 \%$ ); fertility control and culling (6 papers, $15 \%$ ); and fertility control and movement restriction (1 paper, $3 \%$ ) (Table 2$)$. Of the papers that involved fertility control, $79 \%$ (23 of 29 papers) controlled the fertility of both male and female dogs (Tables 3 and 4). Eight papers (21\%) directly compared different methods of management: three compared fertility control and culling $(8 \%)$; three compared fertility control and sheltering $(8 \%)$; one compared fertility control and movement restriction (3\%); and one compared different taxation methods ( $3 \%)$.

\subsubsection{Impact Category and Indicators of Effect}

Dog population management methods were investigated in terms of the impact they have on: dog health and welfare (6 papers, $15 \%$ ); dog demographics (13 papers, 33\%); public attitude to free-roaming populations ( 3 papers, $8 \%$ ); public health (16 papers, $41 \%$ ); and risk to wildlife populations (1 paper, $3 \%$ ) (Table S3). To evaluate these impacts, the final corpus reported 19 different indicators of effect.

The majority of these were different indicators of dog health and welfare, and public health risk, and relatively few different indicators were used to assess dog demographics and public attitude. Considering all the reported indicators, studies used dog population size most frequently to evaluate impact (19 papers, 49\%). Considering all management methods and indicators, studies most often evaluated the effect of fertility control and sheltering using dog population size as an indicator (8 papers, $21 \%$ ).

\subsection{Quality Evaluation}

We assessed the quality of the intervention and observational studies in the final corpus. We split our measures into two categories: those that applied to all papers (including study design, reporting of aims/hypotheses, appropriate outcome studied, and definition of study population), and those that applied to papers depending on their study design (inclusion of power calculation, sample size calculation, control population, inter-observer reliability, and reporting of baseline characteristics).

\subsubsection{Study Design and Study Populations}

In the final corpus, 33 papers used only one study design and six papers used two different study designs within the paper (Table 2). Papers in the final corpus used observational (i.e., observing dog population management, but not imposing the intervention themselves) (18 papers, $46 \%$ ), intervention ( 1 paper, $3 \%$ ), modelling (14 papers, $36 \%$ ), a combination of observational study designs ( 3 papers, $8 \%$ ), and a combination of observational and modelling study designs (3 papers, $8 \%$ ). Of the observational study designs, seven papers used a retrospective cohort (33\%), six papers used a longitudinal cross-sectional (29\%), nine papers used a single time point cross-sectional approach (38\%), two papers combined prospective cohort and retrospective cohort $(10 \%)$, and one paper combined a single time point cross-sectional and retrospective cohort study design (5\%). Papers reported various combinations of dog populations, including: free-roaming owned, free-roaming unowned, restricted owned, and shelter dogs (Table 2). Of the various combinations, 36 papers (92\%) investigated both free-roaming unowned and free-roaming owned dogs. Twenty-six of these papers $(72 \%)$ grouped this population as one (e.g., the free-roaming dog population) and did not distinguish between owned and unowned dogs (Table S4). Two papers did not define their study population (5\%). 
Table 1. Impact categories and indicators of effect used in the final corpus to evaluate the effects of management methods. Study design is indicated with either O/I indicating an observational or intervention study, or $\mathrm{M}$ for a modelling study. Following the indication of study design is the number of papers (denoted $=n$, where $\mathrm{n}$ is the number of papers) adopting this design to test this combination of dog population management method, indicator, and measured impact, followed by the reference details for the relevant papers.

\begin{tabular}{|c|c|c|c|c|c|c|c|c|}
\hline Impact & Indicators & $\begin{array}{l}\text { Fertility } \\
\text { Control }\end{array}$ & Culling & Sheltering & Taxation & $\begin{array}{l}\text { Fertility } \\
\text { Control and } \\
\text { Sheltering }\end{array}$ & $\begin{array}{l}\text { Fertility } \\
\text { Control and } \\
\text { Culling }\end{array}$ & $\begin{array}{l}\text { Fertility Control } \\
\text { and Movement } \\
\text { Restriction }\end{array}$ \\
\hline \multirow{7}{*}{$\begin{array}{l}\text { Dog health and } \\
\text { welfare }\end{array}$} & Body condition score & $\begin{array}{l}\mathrm{O} / \mathrm{I}=3 \\
{[50,70,71]}\end{array}$ & & & & & & \\
\hline & Measure of dog behaviour & & & $\begin{array}{l}\mathrm{O} / \mathrm{I}=1 \\
{[80]}\end{array}$ & & & & \\
\hline & $\begin{array}{l}\text { Physiological stress } \\
\text { measures }\end{array}$ & & & & & $\mathrm{O} / \mathrm{I}=1[81]$ & & \\
\hline & Presence of injury & $\mathrm{O} / \mathrm{I}=1[70]$ & & & & & & \\
\hline & Visible skin condition & $\mathrm{O} / \mathrm{I}=2[50,71]$ & & & & & & \\
\hline & $\begin{array}{l}\text { Dog disease prevalence } \\
\text { (ectoparasites, viruses or } \\
\text { bacterial infection) }\end{array}$ & $\mathrm{O} / \mathrm{I}=1[70]$ & & & & & & \\
\hline & $\begin{array}{l}\text { Fertility control related } \\
\text { complications }\end{array}$ & $\mathrm{O} / \mathrm{I}=1$ [82] & & & & & & \\
\hline $\begin{array}{l}\text { Dog population } \\
\text { demographics }\end{array}$ & Dog population size & $\begin{array}{l}\mathrm{O} / \mathrm{I}=4 \\
{[68,69,83,84]} \\
\mathrm{M}=7[69,85-90]\end{array}$ & $\mathrm{M}=3[85,88,91]$ & $\begin{array}{l}M=2 \\
{[87,90]}\end{array}$ & $\begin{array}{l}M=1 \\
{[92]}\end{array}$ & $\begin{array}{l}\mathrm{O} / \mathrm{I}=5 \\
{[67,73,93-95]}\end{array}$ & & $\mathrm{M}=3[87,89,96]$ \\
\hline Public attitude & $\begin{array}{l}\text { Public attitude towards } \\
\text { free-roaming dogs }\end{array}$ & $\mathrm{O} / \mathrm{I}=1[97]$ & & & & $\mathrm{O} / \mathrm{I}=1[94]$ & & \\
\hline
\end{tabular}


Table 1. Cont

\begin{tabular}{|c|c|c|c|c|c|c|c|c|}
\hline Impact & Indicators & $\begin{array}{l}\text { Fertility } \\
\text { Control }\end{array}$ & Culling & Sheltering & Taxation & $\begin{array}{l}\text { Fertility } \\
\text { Control and } \\
\text { Sheltering }\end{array}$ & $\begin{array}{l}\text { Fertility } \\
\text { Control and } \\
\text { Culling }\end{array}$ & $\begin{array}{c}\text { Fertility Control } \\
\text { and Movement } \\
\text { Restriction }\end{array}$ \\
\hline \multirow{10}{*}{$\begin{array}{l}\text { Public health } \\
\text { risk }\end{array}$} & $\begin{array}{l}\text { Number of human rabies } \\
\text { cases }\end{array}$ & $\begin{array}{l}\mathrm{O} / \mathrm{I}=2[68,72] \\
\mathrm{M}=1[98]\end{array}$ & & & & $\mathrm{O} / \mathrm{I}=1[95]$ & & \\
\hline & Human bite cases & $\mathrm{O} / \mathrm{I}=1[99]$ & & & & & & \\
\hline & Dog rabies prevalence & $M=1[100]$ & $M=3[100-102]$ & & & & & \\
\hline & $\begin{array}{l}\text { Echinococcus granulosus } \\
\text { prevalence in humans }\end{array}$ & & & $\mathrm{O} / \mathrm{I}=1[5]$ & & & $\mathrm{O} / \mathrm{I}=1[103]$ & \\
\hline & $\begin{array}{l}\text { Echinococcus granulosus } \\
\text { prevalence in livestock }\end{array}$ & & & $\mathrm{O} / \mathrm{I}=1[5]$ & & & $\begin{array}{l}\mathrm{O} / \mathrm{I}=2 \\
{[103,104]}\end{array}$ & \\
\hline & $\begin{array}{l}\text { Echinococcus granulosus } \\
\text { prevalence in dogs }\end{array}$ & & & $\mathrm{O} / \mathrm{I}=1[5]$ & & & $\begin{array}{l}\mathrm{O} / \mathrm{I}=2 \\
{[103,104]}\end{array}$ & \\
\hline & $\begin{array}{l}\text { Dog disease prevalence } \\
\text { (visible skin conditions, } \\
\text { ectoparasites, viruses or } \\
\text { bacterial infection) }\end{array}$ & & & & & $\mathrm{O} / \mathrm{I}=1[93]$ & & \\
\hline & $\begin{array}{l}\text { Prevalence of visceral } \\
\text { leishmaniasis in dogs }\end{array}$ & & $\mathrm{O} / \mathrm{I}=2[105,106]$ & & & & & \\
\hline & $\begin{array}{l}\text { Prevalence of visceral } \\
\text { leishmaniasis in children }\end{array}$ & & $\mathrm{O} / \mathrm{I}=1[106]$ & & & & & \\
\hline & Rabies R0 & & $\begin{array}{l}M=3 \\
{[91,107,108]}\end{array}$ & & & & & \\
\hline $\begin{array}{l}\text { Risk to wildlife } \\
\text { populations }\end{array}$ & $\begin{array}{l}\text { Canine distemper } \\
\text { prevalence in wildlife } \\
\text { populations }\end{array}$ & $M=1[109]$ & & & & & & \\
\hline
\end{tabular}


Table 2. All final corpus papers by management factors (method and intensity), study design factors, and reporting quality. Management intensity is reported in terms of coverage and length. Length is reported as years of: (i) mgmt. = management (indicating the study and management method took place at the same time) or (ii) study (indicating the study took place after management began). NA = not applicable for the study design.

\begin{tabular}{|c|c|c|c|c|c|c|c|}
\hline Paper & $\begin{array}{l}\text { Dog Population } \\
\text { Management } \\
\text { Method }\end{array}$ & $\begin{array}{l}\text { Management Intensity: } \\
\text { Coverage (C) and Length } \\
\text { (L) of Management/Study }\end{array}$ & Dog Population Type & Study Design & $\begin{array}{c}\text { No. } \\
\text { Replicates }\end{array}$ & No. Groups & $\begin{array}{c}\text { Reporting } \\
\text { Quality Indicator } \\
\text { Score }\end{array}$ \\
\hline [91] & Culling & Up to $33 \%$ & $\begin{array}{l}\text { Free-roaming stray, Free-roaming } \\
\text { owned, Restricted owned }\end{array}$ & Modelling & NA & NA & NA \\
\hline [101] & Culling & Various & $\begin{array}{l}\text { Free-roaming stray, Free-roaming } \\
\text { owned, Restricted owned }\end{array}$ & Modelling & NA & NA & NA \\
\hline [102] & Culling & $5 \%$ and $10 \%$ & Undefined & Modelling & NA & NA & NA \\
\hline [107] & Culling & Various & $\begin{array}{l}\text { Free-roaming stray, Free-roaming } \\
\text { owned, Restricted owned }\end{array}$ & Modelling & NA & NA & NA \\
\hline [108] & Culling & Various & Undefined & Modelling & NA & NA & NA \\
\hline [106] & Culling & $\begin{array}{l}\text { C: Not reported } \\
\text { L: first } 2 \text { years mgmt }\end{array}$ & $\begin{array}{l}\text { Free-roaming stray, Free-roaming } \\
\text { owned }\end{array}$ & Intervention & 1 & $\begin{array}{l}2 \text { (management and } \\
\text { control) }\end{array}$ & $50 \%(2 / 4)$ \\
\hline [105] & Culling & $\begin{array}{c}\text { C: } 8 \% \\
\text { L: } 14 \text { months study }\end{array}$ & $\begin{array}{l}\text { Free-roaming stray, Free-roaming } \\
\text { owned }\end{array}$ & Observational-cross-sectional-longitudinal & 1 & 1 & $20 \%(1 / 4)$ \\
\hline$[86]$ & Fertility control & Various ( $65 \%$ and above) & $\begin{array}{l}\text { Free-roaming stray, Free-roaming } \\
\text { owned, Restricted owned }\end{array}$ & $\begin{array}{c}\text { Modelling and } \\
\text { Observational-cross-sectional-single time } \\
\text { point }\end{array}$ & NA & NA & NA \\
\hline [98] & Fertility control & 25 to $50 \%$ & $\begin{array}{l}\text { Free-roaming stray, Free-roaming } \\
\text { owned, Restricted owned }\end{array}$ & Modelling & NA & NA & NA \\
\hline [109] & Fertility control & $\begin{array}{c}\text { Simulate a } 50 \%, 75 \% \text { and } \\
90 \% \text { reduction, but do not } \\
\text { specify what neutering rate } \\
\text { would achieve this }\end{array}$ & $\begin{array}{l}\text { Free-roaming stray, Free-roaming } \\
\text { owned }\end{array}$ & Modelling & NA & NA & NA \\
\hline [97] & Fertility control & $\begin{array}{c}\text { C: NR } \\
\text { L: } 3 \text { years study }\end{array}$ & $\begin{array}{l}\text { Free-roaming stray, Free-roaming } \\
\text { owned, Restricted owned }\end{array}$ & Observational-cross-sectional-longitudinal & 1 & 1 & $80 \%(4 / 5)$ \\
\hline [84] & Fertility control & $\begin{array}{c}\text { C: } 15 \% \text { of males and } 31 \% \text { of } \\
\text { females } \\
\text { L: } 1.5 \text { years mgmt }\end{array}$ & $\begin{array}{l}\text { Owned (free-roaming), Owned } \\
\text { (restricted) }\end{array}$ & $\begin{array}{l}\text { Observational-cross-sectional-single time } \\
\text { point }\end{array}$ & 1 & 1 & $50 \%(1 / 2)$ \\
\hline [82] & Fertility control & C/L: NA & $\begin{array}{l}\text { Free-roaming stray, Free-roaming } \\
\text { owned }\end{array}$ & $\begin{array}{l}\text { Observational-cohort-prospective and } \\
\text { Observational-cohort-retrospective }\end{array}$ & 1 & 1 & $40 \%(2 / 5)$ \\
\hline [99] & Fertility control & $\begin{array}{l}\text { C: } 65 \% \text { of females } \\
\text { L: Not reported }\end{array}$ & $\begin{array}{l}\text { Free-roaming stray, Free-roaming } \\
\text { owned }\end{array}$ & Observational-cohort-retrospective & 1 & 1 & $40 \%(2 / 5)$ \\
\hline
\end{tabular}


Table 2. Cont.

\begin{tabular}{|c|c|c|c|c|c|c|c|}
\hline Paper & $\begin{array}{l}\text { Dog Population } \\
\text { Management } \\
\text { Method }\end{array}$ & $\begin{array}{l}\text { Management Intensity: } \\
\text { Coverage (C) and Length } \\
\text { (L) of Management/Study }\end{array}$ & Dog Population Type & Study Design & $\begin{array}{c}\text { No. } \\
\text { Replicates }\end{array}$ & No. Groups & $\begin{array}{c}\text { Reporting } \\
\text { Quality Indicator } \\
\text { Score }\end{array}$ \\
\hline$[70]$ & Fertility control & $\begin{array}{c}\text { C: } \sim 80 \% \text { of females } \\
\text { L: Various }-17,7 \text {, and } 0 \\
\text { years mgmt }\end{array}$ & $\begin{array}{l}\text { Free-roaming stray, Free-roaming } \\
\text { owned }\end{array}$ & $\begin{array}{l}\text { Observational-cross-sectional-single time } \\
\text { point }\end{array}$ & 1 & $\begin{array}{l}3 \text { (2 CNR intensities } \\
\text { and a control) }\end{array}$ & $25 \%(1 / 4)$ \\
\hline [69] & Fertility control & $\begin{array}{l}\text { C: } 62 \text { to } 87 \% \\
\text { L: } 2 \text { years } \mathrm{mg}\end{array}$ & $\begin{array}{l}\text { Free-roaming stray, Free-roaming } \\
\text { owned }\end{array}$ & $\begin{array}{l}\text { Observational-cross-sectional-longitudinal } \\
\text { and Modelling }\end{array}$ & 6 & 1 & $20 \%(1 / 5)$ \\
\hline$[50]$ & Fertility control & $\begin{array}{l}\text { C: Not reported } \\
\text { L: } 2 \text { years mgmt }\end{array}$ & $\begin{array}{l}\text { Free-roaming stray, Free-roaming } \\
\text { owned }\end{array}$ & $\begin{array}{l}\text { Observational-cross-sectional-single time } \\
\text { point }\end{array}$ & 1 & 2 (CNR and control) & $100 \%(3 / 3)$ \\
\hline$[83]$ & Fertility control & $\begin{array}{l}\text { C: Not reported } \\
\text { L: } 12 \text { years study }\end{array}$ & $\begin{array}{l}\text { Free-roaming stray, Free-roaming } \\
\text { owned }\end{array}$ & Observational-cross-sectional-longitudinal & 1 & 1 & $0 \%(0 / 3)$ \\
\hline$[68]$ & Fertility control & $\begin{array}{l}\text { C: } 65 \% \text { of females } \\
\text { L: } 8 \text { years mgmt }\end{array}$ & $\begin{array}{l}\text { Free-roaming stray, Free-roaming } \\
\text { owned }\end{array}$ & $\begin{array}{l}\text { Observational-cross-sectional-longitudinal } \\
\text { and Observational-cohort-retrospective }\end{array}$ & 1 & 1 & $0 \%(0 / 4)$ \\
\hline$[71]$ & Fertility control & $\begin{array}{c}\text { C: } 61 \% \\
\text { L: } 2 \text { years mgmt }\end{array}$ & $\begin{array}{l}\text { Free-roaming stray, Free-roaming } \\
\text { owned }\end{array}$ & $\begin{array}{l}\text { Observational-cross-sectional-single time } \\
\text { point }\end{array}$ & 1 & 2 (CNR and control) & $0 \%(0 / 1)$ \\
\hline$[85]$ & $\begin{array}{l}\text { Fertility control } \\
\text { and culling }\end{array}$ & Various & $\begin{array}{l}\text { Free-roaming stray, Free-roaming } \\
\text { owned }\end{array}$ & Modelling & NA & NA & NA \\
\hline [88] & $\begin{array}{l}\text { Fertility control } \\
\text { and culling }\end{array}$ & Various & Free-roaming stray & Modelling & NA & NA & NA \\
\hline$[100]$ & $\begin{array}{l}\text { Fertility control } \\
\text { and culling }\end{array}$ & Various & $\begin{array}{l}\text { Free-roaming stray, Free-roaming } \\
\text { owned }\end{array}$ & Modelling & NA & NA & NA \\
\hline$[72]$ & $\begin{array}{l}\text { Fertility control } \\
\text { and culling }\end{array}$ & $\begin{array}{l}\text { C: Fertility control } 3 \% \\
\text { (max). Culling } 10 \% \\
\text { L: } 30 \text { years study }\end{array}$ & $\begin{array}{l}\text { Free-roaming stray, Free-roaming } \\
\text { owned }\end{array}$ & Observational-cohort-retrospective & 1 & 1 & $25 \%(1 / 4)$ \\
\hline [103] & $\begin{array}{l}\text { Fertility control } \\
\text { and culling }\end{array}$ & $\begin{array}{l}\text { C: Not reported } \\
\text { L: } 8 \text { years mgmt }\end{array}$ & Free-roaming stray & Observational-cohort-retrospective & 1 & 1 & $20 \%(1 / 4)$ \\
\hline [104] & $\begin{array}{l}\text { Fertility control } \\
\text { and culling }\end{array}$ & $\begin{array}{l}\text { C: Fertility control: } 8 \% . \\
\text { Culling: } 67 \% \\
\text { L: } 4 \text { years mgmt }\end{array}$ & Free-roaming stray & Observational-cross-sectional-longitudinal & 1 & 1 & $20 \%(1 / 4)$ \\
\hline [89] & $\begin{array}{l}\text { Fertility control } \\
\text { and movement } \\
\text { restriction }\end{array}$ & Various & Free-roaming owned & $\begin{array}{c}\text { Modelling and } \\
\text { Observational-cross-sectional-single time } \\
\text { point }\end{array}$ & NA & NA & NA \\
\hline$[87]$ & $\begin{array}{l}\text { Fertility control } \\
\text { and sheltering }\end{array}$ & Various & $\begin{array}{c}\text { Free-roaming stray, Free-roaming } \\
\text { owned, Restricted owned, Shelter } \\
\text { dogs }\end{array}$ & Modelling & NA & NA & NA \\
\hline
\end{tabular}


Table 2. Cont.

\begin{tabular}{|c|c|c|c|c|c|c|c|}
\hline Paper & $\begin{array}{l}\text { Dog Population } \\
\text { Management } \\
\text { Method }\end{array}$ & $\begin{array}{l}\text { Management Intensity: } \\
\text { Coverage (C) and Length } \\
\text { (L) of Management/Study }\end{array}$ & Dog Population Type & Study Design & $\begin{array}{l}\text { No. } \\
\text { Replicates }\end{array}$ & No. Groups & $\begin{array}{l}\text { Reporting } \\
\text { Quality Indicator } \\
\text { Score }\end{array}$ \\
\hline [90] & $\begin{array}{l}\text { Fertility control } \\
\text { and sheltering }\end{array}$ & $\begin{array}{l}\text { CNR: } 20-40 \% \text { more } \\
\text { captures. Sheltering: } 10 \% \\
\text { increase. }\end{array}$ & $\begin{array}{l}\text { Free-roaming stray, Free-roaming } \\
\text { owned, Restricted owned, Shelter } \\
\text { dogs }\end{array}$ & Modelling & NA & NA & NA \\
\hline [96] & $\begin{array}{c}\text { Fertility control } \\
\text { and sheltering }\end{array}$ & $\begin{array}{l}\text { Various (from } 0 \text { up to } 0.2 \\
\text { per year) }\end{array}$ & $\begin{array}{l}\text { Free-roaming stray, Free-roaming } \\
\text { owned }\end{array}$ & Modelling & NA & NA & NA \\
\hline [73] & $\begin{array}{l}\text { Fertility control } \\
\text { and sheltering }\end{array}$ & $\begin{array}{c}\text { C: } 88 \% \\
\text { L: } 14 \text { months study }\end{array}$ & $\begin{array}{l}\text { Free-roaming stray, Free-roaming } \\
\text { owned }\end{array}$ & Observational-cross-sectional-longitudinal & 1 & $\begin{array}{l}2 \text { (management and } \\
\text { control) }\end{array}$ & $67 \%(2 / 3)$ \\
\hline [94] & $\begin{array}{l}\text { Fertility control } \\
\text { and sheltering }\end{array}$ & C/L: Not reported & $\begin{array}{l}\text { Free-roaming stray, Free-roaming } \\
\text { owned }\end{array}$ & $\begin{array}{l}\text { Observational-cross-sectional-single time } \\
\text { point }\end{array}$ & 1 & 1 & $20 \%(1 / 4)$ \\
\hline [93] & $\begin{array}{l}\text { Fertility control } \\
\text { and sheltering }\end{array}$ & $\begin{array}{l}\text { C: Fertility control: } 43 \% . \\
\text { Sheltered: } 33 \% \\
\text { L: } 9 \text { months mgmt }\end{array}$ & $\begin{array}{l}\text { Free-roaming stray, Free-roaming } \\
\text { owned, Restricted owned }\end{array}$ & $\begin{array}{l}\text { Observational-cohort-prospective and } \\
\text { Observational-cohort-retrospective }\end{array}$ & 1 & 1 & $20 \%(1 / 4)$ \\
\hline [67] & $\begin{array}{l}\text { Fertility control } \\
\text { and sheltering }\end{array}$ & $\begin{array}{l}\text { C: Not reported } \\
\text { L: } 13 \text { years mgmt }\end{array}$ & $\begin{array}{l}\text { Free-roaming stray, Free-roaming } \\
\text { owned }\end{array}$ & Observational-cohort-retrospective & 1 & 1 & $0 \%(0 / 3)$ \\
\hline [95] & $\begin{array}{l}\text { Fertility control } \\
\text { and sheltering }\end{array}$ & $\begin{array}{l}\text { C: Fertility control: } \\
\text { between } 0.03 \text { to } 12 \% \text {. } \\
\text { Sheltering: NR } \\
\text { L: } 5 \text { years study }\end{array}$ & $\begin{array}{l}\text { Free-roaming stray, Free-roaming } \\
\text { owned }\end{array}$ & Observational-cohort-retrospective & 1 & 1 & $0 \%(0 / 2)$ \\
\hline [81] & $\begin{array}{c}\text { Fertility control } \\
\text { and sheltering }\end{array}$ & C/L: NA & Free-roaming stray & $\begin{array}{l}\text { Observational-cross-sectional-single time } \\
\text { point }\end{array}$ & 1 & 1 & $0 \%(0 / 3)$ \\
\hline [80] & Sheltering & C/L: NA & Free-roaming stray & $\begin{array}{c}\text { Observational-cross-sectional-single time } \\
\text { point and } \\
\text { Observational-cohort-retrospective }\end{array}$ & 1 & $\begin{array}{l}2 \text { (previously unowned } \\
\text { free-roaming; } \\
\text { previously owned) }\end{array}$ & $0 \%(0 / 3)$ \\
\hline [5] & Sheltering & $\begin{array}{l}\text { C: Not reported } \\
\text { L: } 11 \text { years mgmt }\end{array}$ & Free-roaming stray, Shelter dogs & Observational-cohort-retrospective & 1 & 1 & $0 \%(0 / 4)$ \\
\hline [92] & Taxation & NA & $\begin{array}{c}\text { Free-roaming stray, Free-roaming } \\
\text { owned, Restricted owned, Shelter } \\
\text { dogs }\end{array}$ & Modelling & NA & NA & NA \\
\hline
\end{tabular}


Table 3. Results from papers in the final corpus (excluding modelling studies) of the effects of methods of dog population management on the indicators of impact and impact categories. $\uparrow$ indicates an increasing effect, $\downarrow$ a decreasing effect and n.e. no effect; combinations of different symbols indicate where evidence is conflicting. Where $p$-values were reported, this is included (e.g., $p<0.05$ ), NR $=p$-value was not reported, NS $=p$-value not significant. NA $=$ not applicable for the study design. The size of effect is extracted from papers and reported in terms of the years of: (i) mgmt. = management (indicating the study and management method took place at the same time) or (ii) study (indicating the study took place after management began). Where fertility control is included in the dog population management method, $(\mathrm{M} \& \mathrm{~F})$ indicates fertility control was applied to both males and females, (F) indicates only female fertility was controlled. Supporting evidence is provided in references.

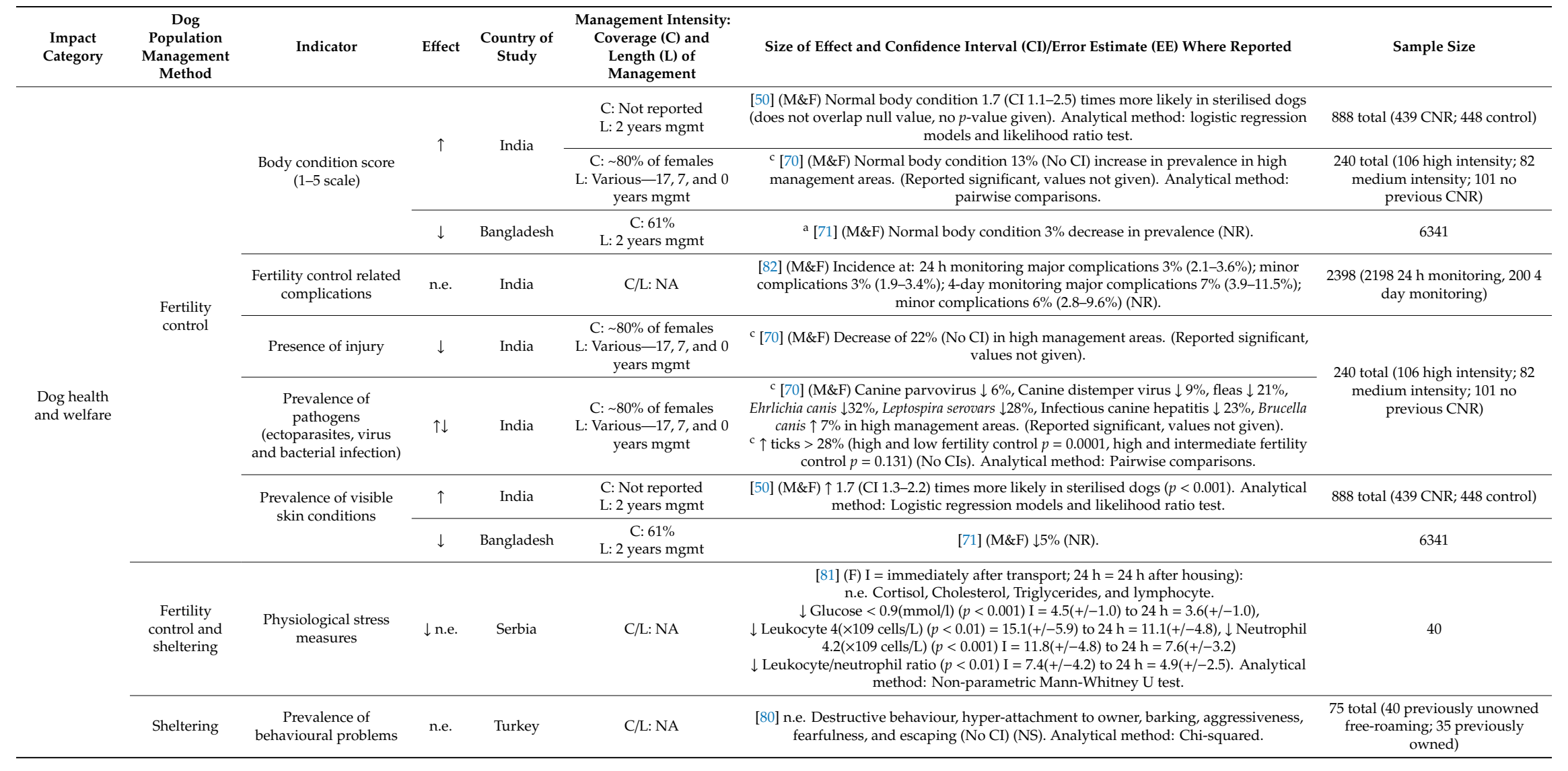


Table 3. Cont

\begin{tabular}{|c|c|c|c|c|c|c|c|}
\hline $\begin{array}{l}\text { Impact } \\
\text { Category }\end{array}$ & $\begin{array}{l}\text { Dog } \\
\text { Population } \\
\text { Management } \\
\text { Method } \\
\end{array}$ & Indicator & Effect & $\begin{array}{l}\text { Country of } \\
\text { Study }\end{array}$ & $\begin{array}{l}\text { Management Intensity: } \\
\text { Coverage (C) and } \\
\text { Length (L) of } \\
\text { Management }\end{array}$ & Size of Effect and Confidence Interval (CI)/Error Estimate (EE) Where Reported & Sample Size \\
\hline \multirow{8}{*}{$\begin{array}{l}\text { Dog } \\
\text { population } \\
\text { demograph-ics }\end{array}$} & \multirow{3}{*}{$\begin{array}{l}\text { Fertility } \\
\text { control }\end{array}$} & \multirow{3}{*}{ Dog population size } & \multirow{2}{*}{$\downarrow$} & India & $\begin{array}{l}\text { C: Not reported } \\
\text { L: } 12 \text { years study }\end{array}$ & [83] (M\&F) $\downarrow \sim 40 \%$ b (NR). & $\mathrm{NA}$ \\
\hline & & & & Brazil & $\begin{array}{c}\text { C: } 15 \% \text { of males and } 31 \% \\
\text { of females } \\
\text { L: } 1.5 \text { years } \mathrm{mgmt}\end{array}$ & [84] (M\&F) $\downarrow 12 \%$ (NR). & NA \\
\hline & & & $\downarrow$ n.e. & India & $\begin{array}{c}\text { C: } 62 \text { to } 87 \% \\
\text { L: } 2 \text { years mgmt }\end{array}$ & $\begin{array}{l}\text { [69] (M\&F) Both } \downarrow \text { n.e. Decrease between } 3 \%(p>0.05) \text { and } 51 \%(p<0.05) \text {. Analytical } \\
\text { method: Not reported. }\end{array}$ & NA \\
\hline & \multirow{5}{*}{$\begin{array}{l}\text { Fertility } \\
\text { control and } \\
\text { sheltering }\end{array}$} & \multirow{5}{*}{ Dog population size } & \multirow[b]{2}{*}{ n.e. } & Italy & $\begin{array}{l}\text { C: Not reported } \\
\text { L: } 13 \text { years mgmt }\end{array}$ & [67] (M\&F) No effect (NR). & NA \\
\hline & & & & Brazil & $\begin{array}{l}\text { C: } 88 \% \\
\text { L: } 14 \text { months study }\end{array}$ & $\begin{array}{l}\text { [73] (M\&F) No effect (NR). Control (area A): from } 81 \text { (66-97) to } 94 \text { (75-113). Intervention } \\
\text { (area B): from } 70 \text { (57-84) to } 81 \text { (65-96). Analytical method: Jolly-Seber mark-recapture } \\
\text { model. }\end{array}$ & NA \\
\hline & & & \multirow{3}{*}{$\downarrow$} & Canada & $\begin{array}{l}\text { C: Fertility control: } 43 \% \text {. } \\
\text { Sheltered: } \\
32 \% \\
\text { L: } 9 \text { months mgmt }\end{array}$ & [93] (M\&F) $\downarrow 34 \%(p<0.001)$. Analytical method: Not reported. & NA \\
\hline & & & & & C/L: Not reported & [94] (M\&F) no quantitative data. & 18 \\
\hline & & & & Thailand & $\begin{array}{l}\text { C: Fertility control: } \\
\text { between } 0.03 \text { to } 12 \% . \\
\text { Sheltering: NR } \\
\text { L: } 5 \text { years study }\end{array}$ & [95] (M\&F) $\downarrow 23 \%$ (NR). & NA \\
\hline \multirow{2}{*}{ Public attitude } & $\begin{array}{l}\text { Fertility } \\
\text { control }\end{array}$ & $\begin{array}{l}\text { Public attitude towards } \\
\text { perception of dog } \\
\text { management method }\end{array}$ & n.e. & Brazil & $\begin{array}{l}\text { C: NR } \\
\text { L: } 3 \text { years study }\end{array}$ & [97] (M\&F) n.e. $(p=0.774)$ (No CI). Analytical method: Chi-squared. & $\begin{array}{l}354 \text { Pre-management; } 70 \\
\text { post-management }\end{array}$ \\
\hline & $\begin{array}{l}\text { Fertility } \\
\text { control and } \\
\text { sheltering }\end{array}$ & $\begin{array}{l}\text { Public attitude towards } \\
\text { free-roaming dogs }\end{array}$ & $\downarrow$ & Canada & C/L: Not reported & [94] (M\&F) No quantitative data. & 18 \\
\hline \multirow{4}{*}{$\begin{array}{l}\text { Public health } \\
\text { risk }\end{array}$} & \multirow{4}{*}{ Culling } & \multirow{3}{*}{$\begin{array}{l}\text { Prevalence of visceral } \\
\text { leishmaniasis in dogs }\end{array}$} & \multirow[b]{2}{*}{$\downarrow$} & \multirow[b]{2}{*}{ Brazil } & $\begin{array}{c}\text { C: } 8 \% \\
\text { L: } 14 \text { months study }\end{array}$ & [105] $\downarrow$ Between $66 \%$ and $69 \%$ (NR). & 328 \\
\hline & & & & & $\begin{array}{l}\text { C: Not reported } \\
\text { L: first } 2 \text { years mgmt }\end{array}$ & $\begin{array}{l}\text { [106] Short term: Initial decrease of } \downarrow 26 \%(p<0.001) \text {. Analytical method: Chi-squared } \\
\text { (temporal changes within areas (intervention and control), and Poisson regression for } \\
\text { between intervention and control. }\end{array}$ & \multirow{2}{*}{$\begin{array}{l}\text { Intervention area: } 1989-1990= \\
235 ; 1990-1991=248 ; 1991-1992 \\
=70 ; 1992-1993=131 ; \text { and } 1993= \\
\text { 164. Control area }=\text { not reported }\end{array}$} \\
\hline & & & n.e. & Brazil & $\begin{array}{l}\text { C: Not reported } \\
\text { L: } 4 \text { years mgmt }\end{array}$ & $\begin{array}{l}\text { [106] Long term: incidence not significantly different between intervention and control } \\
\qquad(p=0.07) \text {. Analytical method: As above. }\end{array}$ & \\
\hline & & $\begin{array}{l}\text { Prevalence of visceral } \\
\text { leishmaniasis in } \\
\text { children }\end{array}$ & $\downarrow$ & Brazil & $\begin{array}{l}\text { C: Not reported. } \\
\text { L: } 4 \text { years mgmt }\end{array}$ & $\begin{array}{c}\text { [106] } \downarrow \text { incidence from } 12 \text { cases/1000 inhabitants/year to } 2 \text { cases/1000 inhabitants/year ( } p \\
<0.01 \text { ). Analytical method: As above. }\end{array}$ & NA \\
\hline
\end{tabular}


Table 3. Cont.

\begin{tabular}{|c|c|c|c|c|c|c|c|}
\hline $\begin{array}{l}\text { Impact } \\
\text { Category }\end{array}$ & $\begin{array}{l}\text { Dog } \\
\text { Population } \\
\text { Management } \\
\text { Method }\end{array}$ & Indicator & Effect & $\begin{array}{l}\text { Country of } \\
\text { Study }\end{array}$ & $\begin{array}{l}\text { Management Intensity: } \\
\text { Coverage (C) and } \\
\text { Length (L) of } \\
\text { Management }\end{array}$ & Size of Effect and Confidence Interval (CI)/Error Estimate (EE) Where Reported & Sample Size \\
\hline \multirow{13}{*}{$\begin{array}{l}\text { Public health } \\
\text { risk }\end{array}$} & \multirow{2}{*}{$\begin{array}{l}\text { Fertility } \\
\text { control }\end{array}$} & Human bite cases & $\downarrow$ & India & $\begin{array}{l}\text { C: } 65 \% \text { of females } \\
\text { L: Not reported }\end{array}$ & $\begin{array}{c}\mathrm{b}[99] \text { (F) } \downarrow 5 \text { bites per month }(p<0.001)^{\mathrm{b}} \text {. Analytical method: Linear least squares } \\
\text { regression. }\end{array}$ & NA \\
\hline & & $\begin{array}{l}\text { Number of human } \\
\text { rabies cases }\end{array}$ & $\downarrow$ & India & $\begin{array}{l}\text { C: } 65 \% \text { of females. } \\
\text { L: } 10 \text { years mgmt }\end{array}$ & [68] (M\&F) $\downarrow 100 \%$ (NR). & NA \\
\hline & \multirow{6}{*}{$\begin{array}{l}\text { Fertility } \\
\text { control and } \\
\text { culling }\end{array}$} & $\begin{array}{l}\text { Number of human } \\
\text { rabies cases }\end{array}$ & $\downarrow$ & Sri Lanka & $\begin{array}{l}\text { C: Fertility control } 3 \% \\
\text { (max). Culling } 10 \% \\
\text { L: } 30 \text { years study }\end{array}$ & [72] (M\&F) $\downarrow 82 \%$ (NR). & NA \\
\hline & & $\begin{array}{l}\text { Echinococcus granulosus } \\
\text { prevalence in humans }\end{array}$ & n.e. & Cyprus & $\begin{array}{l}\text { C: Not reported } \\
\text { L: } 8 \text { years } \mathrm{mgmt}\end{array}$ & $\begin{array}{l}\text { [103] (F) n.e. on the number of people operated on for Echinococcus granulosus cysts } \\
\text { (NR). }\end{array}$ & NA \\
\hline & & \multirow{2}{*}{$\begin{array}{l}\text { Echinococcus granulosus } \\
\text { prevalence in livestock }\end{array}$} & \multirow[b]{2}{*}{$\downarrow$} & \multirow[b]{2}{*}{ Cyprus } & $\begin{array}{l}\text { C: Not reported } \\
\text { L: } 5 \text { years mgmt }\end{array}$ & $\begin{array}{l}\text { [103] (F) } \downarrow \text { overall infection rate (cattle from } 0.09 \% \text { to } 0.01 \% \text {, sheep from } 0.03 \% \text { to } 0.02 \% \text {, } \\
\text { and goats from } 0.01 \% \text { to } 0.003 \% \text { ) (NR). }\end{array}$ & $\begin{array}{c}1,899,040 \text { total }(104,134 \text { cattle; } \\
885,618 \text { sheep; and } 909,288 \text { goats })\end{array}$ \\
\hline & & & & & $\begin{array}{l}\text { C: Fertility control: } 8 \% . \\
\text { Culling: } 67 \% \\
\text { L: } 4 \text { years mgmt }\end{array}$ & [104] (F) $\downarrow$ prevalence between $47 \%$ to $2 \%$ (depending on species and age) (NR). & Not reported \\
\hline & & \multirow{2}{*}{$\begin{array}{l}\text { Echinococcus granulosus } \\
\text { prevalence in dogs }\end{array}$} & \multirow[b]{2}{*}{$\downarrow$} & \multirow{2}{*}{ Cyprus } & $\begin{array}{l}\text { C: Not reported } \\
\text { L: } 6 \text { years mgmt }\end{array}$ & [103] (F) $\downarrow 100 \%$ in dogs (NR). & 2391 \\
\hline & & & & & $\begin{array}{l}\text { C: Fertility control: } 8 \% \text {. } \\
\text { Culling: } 67 \% \\
\text { L: } 4 \text { years mgmt }\end{array}$ & [104] (F) $\downarrow 80 \%$ in dogs (NR). & 12,213 in 1972; 3947 in 1976 \\
\hline & \multirow[t]{2}{*}{$\begin{array}{l}\text { Fertility } \\
\text { control and } \\
\text { sheltering }\end{array}$} & $\begin{array}{l}\text { Dog disease prevalence } \\
\text { (helminths, Isospora, } \\
\text { Sarcocystis, Giardia, } \\
\text { Cryptosporidium, Taenia, } \\
\text { Echinococcus spp, } \\
\text { Dirofilaria immitis, } \\
\text { Ehrlichia canis, Borrelia } \\
\text { burgdorferi and } \\
\text { Anaplasma } \\
\text { phagocytophilum, and } \\
\text { Toxoplasma gondii) }\end{array}$ & n.e. & Canada & $\begin{array}{c}\text { C: Fertility control: } 43 \% \text {. } \\
\text { Sheltered: } 33 \% \text {. } \\
\text { L: } 9 \text { month mgmt }\end{array}$ & [93] (M\&F) Overall $\downarrow 43 \%(p<0.001)$. Analytical method: Chi-squared. & 145 Pre-clinic; 95 post-clinic \\
\hline & & $\begin{array}{l}\text { Number of human } \\
\text { rabies cases }\end{array}$ & $\downarrow$ & Thailand & $\begin{array}{l}\text { C: Fertility control: } \\
\text { between } 0.03 \text { to } 12 \% . \\
\text { Sheltering: NR. } \\
\text { L: } 6 \text { years study }\end{array}$ & [95] $\downarrow 15 \%$ (NR). & $\mathrm{NA}$ \\
\hline & \multirow{3}{*}{ Sheltering } & $\begin{array}{l}\text { Echinococcus granulosus } \\
\text { prevalence in humans }\end{array}$ & $\downarrow$ & Spain & $\begin{array}{l}\text { C: Not reported } \\
\text { L: } 11 \text { years mgmt }\end{array}$ & [5] $\downarrow 97 \%$ (NR). & $\mathrm{NA}$ \\
\hline & & $\begin{array}{l}\text { Echinococcus granulosus } \\
\text { prevalence in livestock }\end{array}$ & $\downarrow$ & Spain & $\begin{array}{l}\text { C: Not reported } \\
\text { L: } 11 \text { years } \mathrm{mgmt}\end{array}$ & [5] $\downarrow 75 \%$ (NR). & 376 in 1992; 1172 in 1999 \\
\hline & & $\begin{array}{l}\text { Echinococcus granulosus } \\
\text { prevalence in dogs }\end{array}$ & $\downarrow$ & Spain & $\begin{array}{l}\text { C: Not reported } \\
\text { L: } 11 \text { years mgmt }\end{array}$ & [5] $\downarrow 79 \%$ (NR). & 553 in 1989; 1040 in 1998 \\
\hline
\end{tabular}

${ }^{a}$ Contradictory result within paper, contacted author to confirm correct results. ${ }^{b}$ Estimated by approximating numbers from figures in paper. ${ }^{c}$ Alpha value for pairwise post-hoc adjusted to 0.005 to control for multiple comparisons. 
Table 4. Results from only modelling papers from the final corpus of the effects of methods of dog population management on the indicators of impact and impact categories. $\uparrow$ indicates an increasing effect, $\downarrow$ a decreasing effect, and n.e. no effect; combinations of different symbols indicate where evidence is conflicting. The size of effect is extracted from papers and reported in terms of the years of modelling simulation. Supporting evidence is provided in references.

\begin{tabular}{|c|c|c|c|c|c|c|}
\hline $\begin{array}{l}\text { Impact } \\
\text { Category }\end{array}$ & $\begin{array}{l}\text { Dog Population } \\
\text { Management } \\
\text { Method }\end{array}$ & Indicator & Effect & Country of Study & Management Coverage & Size of Effect \\
\hline \multirow{15}{*}{$\begin{array}{l}\text { Dog } \\
\text { population } \\
\text { demographics }\end{array}$} & \multirow{3}{*}{ Culling } & \multirow{3}{*}{ Dog population size } & \multirow{3}{*}{$\downarrow$} & No specific country & Up to $33 \%$ & [91] Decreasing trend. \\
\hline & & & & North America & Various & [88] Decreasing trend. \\
\hline & & & & India & Various & {$[85] * \downarrow 13 \%$ over 20 years. } \\
\hline & \multirow{6}{*}{ Fertility control } & \multirow{6}{*}{ Dog population size } & \multirow{5}{*}{$\downarrow$} & \multirow{2}{*}{ India } & 62 to $87 \%$ & [69] $\downarrow 69 \%$ (80\% neutering coverage) over 20 years. \\
\hline & & & & & Various & {$[85]^{*} \downarrow$ Between $55 \%$ and $75 \%$ over 20 years. } \\
\hline & & & & Brazil & Various (65\% and above) & [86] Decreasing trend. \\
\hline & & & & Mexico & Various & $\begin{array}{l}\text { [89] } \downarrow \text { Between } 14 \% \text { and } 78 \% \text { (depending on neutering effort and } \\
\text { targeting young vs. mixed age dogs) over } 20 \text { years. }\end{array}$ \\
\hline & & & & Italy & $20-40 \%$ more captures. & [90] $\downarrow 34 \%$ over 10 years. \\
\hline & & & n.e. & India & 62 to $87 \%$ & [69] n.e. (31\% neutering coverage) over 20 years. \\
\hline & \multirow[b]{2}{*}{ Sheltering } & \multirow[b]{2}{*}{ Dog population size } & n.e. & North America & Various & [87] n.e. over $30+$ years \\
\hline & & & $\downarrow$ n.e. & Italy & $10 \%$ increase & {$[90] \downarrow 3 \%$ and n.e. over 10 years. } \\
\hline & Taxation & Dog population size & $\downarrow$ & No specific country & NA & [92] Decreasing trend. \\
\hline & \multirow{3}{*}{$\begin{array}{l}\text { Fertility control } \\
\text { and movement } \\
\text { restriction }\end{array}$} & \multirow{3}{*}{ Dog population size } & \multirow{3}{*}{$\downarrow$} & Mexico & Various & $\begin{array}{l}\text { [89] Between }<18 \% \text { and } 73 \% \text { (depending on neutering effort and } \\
\text { confinement level) over } 20 \text { years. }\end{array}$ \\
\hline & & & & Brazil & Various (from 0 up to 0.2 per year) & [96]: $\downarrow 5 \%$ in 30 years. \\
\hline & & & & North America & Various & [87]: Decreasing trend. \\
\hline \multirow{6}{*}{$\begin{array}{l}\text { Public health } \\
\text { risk }\end{array}$} & \multirow{4}{*}{ Culling } & \multirow{2}{*}{ Dog rabies prevalence } & \multirow{2}{*}{$\downarrow$} & $\begin{array}{l}\text { Parameters from } \\
\text { multiple countries }\end{array}$ & Various & {$[100,101]$ Decreasing trend. } \\
\hline & & & & Chad & $5 \%$ and $10 \%$ & [102] Decreasing trend \\
\hline & & \multirow{2}{*}{$\begin{array}{l}\text { Rabies basic reproductive } \\
\text { number (R0) }\end{array}$} & \multirow{2}{*}{$\downarrow$} & China & Various & {$[107,108]$ Decreasing trend. } \\
\hline & & & & No specific country & Up to $33 \%$ & [91] Decreasing trend \\
\hline & \multirow{2}{*}{ Fertility control } & Number of human rabies cases & $\downarrow$ & India & 25 to $50 \%$ & [98] $\downarrow 92 \%$ in 5 years. \\
\hline & & Dog rabies prevalence & $\downarrow$ & Multiple countries & Various & [100] Decreasing trend. \\
\hline Wildlife & Fertility control & $\begin{array}{l}\text { Prevalence of canine distemper } \\
\text { in Indian foxes (Vulpes } \\
\text { bengalensis) }\end{array}$ & $\downarrow$ & India & $\begin{array}{l}\text { Simulate a } 50 \%, 75 \% \text { and } 90 \% \\
\text { reduction, but do not specify what } \\
\text { neutering rate would achieve this }\end{array}$ & $\begin{array}{l}{[109] \downarrow \text { Between } 3 \text { fewer canine distemper spill over events per } 10} \\
\text { years (at } 50 \% \text { population reduction) to } 6 \text { fewer canine distemper } \\
\text { spill over events per } 10 \text { years (at } 90 \% \text { population reduction) }\end{array}$ \\
\hline
\end{tabular}

* Estimated by approximating numbers from figures in paper. 


\subsubsection{Study Reporting Quality Indicators}

All papers in the final corpus reported their aims, with the majority aiming to understand the impact of dog population management as a primary objective and others describing methods of dog population estimation, model development, and guideline development. All papers in the final corpus used an appropriate outcome to measure the effect of dog population management (as defined by Hiby et al. (2017) [16]). In the observational/intervention studies, $35 \%$ of papers did not report the management coverage and $9 \%$ did not report the length of management (Table 2). In general, study quality was low in the observational/intervention papers. Only one study used replication (4\%), only six studies investigated different groups (26\%) and only four included a control population $(17 \%)$. Reporting was low for both power calculations (11\%) (i.e., a calculation to determine statistical power: the probability of correctly rejecting the null hypothesis) and sample size calculations (11\%) (i.e., a calculation to determine the minimum sample size required to answer the study question). However, where appropriate, the reporting of inter-observer reliability $(71 \%)$ and baseline characteristics was high (80\%). Table S5 outlines the results of the reporting quality indicators. Reporting quality (RQ) scores are reported in Table 2.

\subsection{Effects of Management Methods on Impact Categories-Observational and Intervention Studies}

The effects of the different methods of dog population management in observational and intervention studies are summarised in Table 3.

\subsubsection{Dog Health and Welfare}

The impacts of fertility control alone, sheltering alone, and combined fertility control and sheltering were investigated on dog health and welfare in observational studies. No papers in the final corpus investigated the effect of culling or taxation on dog health and welfare.

Fertility control significantly increased body condition score in two of three papers. This was achieved when fertility control was implemented at an unreported coverage level over two years of management ([50] 100\% RQ) and when an $80 \%$ coverage was applied to the female free-roaming dog population over both seven and 17 years of management ([70] 25\% RQ). Fertility control was associated with reduced prevalence of injuries ([70] 25\% RQ: $80 \%$ female coverage over seven and 17 years) and had few associated post-operative complications (between $5 \%$ and $7 \%$, depending on the length of observation) ([82] 40\% RQ). Yoak et al. (2014) ([70] 25\% RQ) reported that fertility control (at an unreported coverage level over two years of management) had varying effects on the prevalence of pathogens, depending on the type of pathogen. This paper compared the prevalence of various pathogens between areas where varying levels of fertility control had been applied. Whilst fertility control significantly decreased viruses and most bacteria, it significantly increased the prevalence of ectoparasites (e.g., Rhipicephalus sanguineus) and Brucella canis over the two years of management. Similarly, Totton et al. (2011) ([50] 100\% RQ; unreported management coverage over two years of management) found that neutered dogs were 1.7 times more likely to have a visible skin condition compared to intact dogs.

One study investigated the impact of sheltering on the post-adoptive welfare of previously free-roaming dogs. This study found no significant differences in the prevalence of behavioural problems following adoption, using the behavioural indicators "destructiveness", "hyperattachment to owner", "fearfulness", "aggressiveness", and "excessive barking" ([80] 0\% RQ).

One paper in the final corpus investigated the impact of combined fertility control and sheltering on dog health and welfare. Radisavljevic et al. (2017) ([81] 0\% RQ) reported that neutering, transport, and housing in a new environment did not have a significant effect on physiological stress measures (Table 3). 


\subsubsection{Dog Population Demographics}

The effects of fertility control and combined fertility control and sheltering on dog population demographics were explored through observational studies. All applied fertility control to both male and female dogs at various intensities (see Table 3) and all reported a reduction in dog population size. Totton et al. (2010) ([69] 20\% RQ) described different results between their study areas. These study areas had various levels of fertility control coverage. In three of their five study areas, they observed a decline in the dog population size $(p<0.05)$ (at 62\%, 66\%, and 67\% coverage), in one they found a decreasing trend $(p>0.05)$, and in one study area they saw no effect of fertility control ( $87 \%$ coverage). Although different results were reported for the impact of fertility control and sheltering, one study reported a significant decrease in population size by $34 \%$ when fertility control and sheltering was applied at $43 \%$ over nine months of management ([93] 20\% RQ). It is important to note that this is a particularly short period of management and these initial results may be the immediate effects of sheltering, rather than fertility control.

\subsubsection{Public Attitude}

The effect of fertility control alone and fertility control and sheltering on public attitude was explored in two papers. Costa et al. (2017) ([97] 80\% RQ) reported no effect of fertility control on the public perception towards the effectiveness of different dog population management methods after three years of fertility control at an unspecified level of coverage. Public attitude, in this study, was quantified using a questionnaire with both open and closed questions. Boey (2017) ([94] 20\% RQ) described a positive improvement of public attitude towards the presence of free-roaming dogs after fertility control and sheltering campaigns at an unspecified level of coverage and length. This was measured using qualitative data collected in interviews and discussion groups.

\subsubsection{Public Health Risk}

The effects of culling, fertility control, sheltering, combined fertility control and culling, and combined fertility control and sheltering on public health risk were explored in observational and intervention studies. Two papers in the final corpus investigated the effect of culling on public health risk. Both reported that culling decreased the prevalence of visceral leishmaniasis in dogs over short-term periods, but did not have a significant effect over long-term periods (at an unreported level of coverage over two years of management ([106] 50\% RQ), and 8\% coverage over 14 months of management ([105] 20\% RQ). One study found that culling significantly decreased the prevalence of visceral leishmaniasis in children (decrease in incidence from 12 cases per 1000 people per year to 2 cases per 1000 people per year, at an unreported coverage level over four years of management) ([106]; 50\% $\mathrm{RQ}$ ). Papers were in agreement that fertility control can reduce public health risk, at the investigated management intensities (see Table 3). Fertility control of $65 \%$ of females over an unspecified length of management was associated with a significant reduction in human bite cases (a decrease of five bites per month) ([99] 50\% RQ). Sheltering at an unspecified level of coverage over 11 years of management was associated with a reduction in Echinococcus granulosus prevalence in humans, livestock, and dogs, but significance was not reported ([5] $0 \% \mathrm{RQ}$ ). The combination of fertility control and culling on public health risk was explored in three observational studies. All studies reported a reduction in Echinococcus granulosus prevalence in dogs and in livestock at the reported management intensities (see Table 3), but did not report significant effects. There was no effect of this management method at an unspecified level of coverage on the number of people operated on for Echinococcus granulosus cysts over eight years of management ([103] 20\% RQ). Combined fertility control and sheltering at various management intensities was associated with a decrease in public health risk. Schurer et al. (2015) ([93] $20 \% \mathrm{RQ}$ ) reported a decrease of $43 \%$ of dog parasite prevalence after nine months of population management intervention at $43 \%$ fertility control and $33 \%$ sheltering coverage. 


\subsection{Effects of Management Methods on Impact Categories-Modelling Studies}

The effects of the different methods of dog population management in modelling studies are presented in Table 4. The effects of methods that are directly compared within the final corpus papers are summarised in Table 5.

Table 5. Summary of methods directly compared within papers. All papers included in the final corpus directly comparing different methods of dog population management used a modelling study design.

\begin{tabular}{|c|c|c|c|c|c|}
\hline $\begin{array}{l}\text { Methods } \\
\text { Being }\end{array}$ & Indicator & Eff & & Evidence & Most Effective Method \\
\hline \multirow{4}{*}{$\begin{array}{l}\text { Fertility control } \\
\text { and culling }\end{array}$} & & Fertility control & Culling & & \\
\hline & \multirow{2}{*}{$\begin{array}{l}\text { Dog population } \\
\text { size }\end{array}$} & \multirow{2}{*}{$\downarrow$} & \multirow{2}{*}{$\downarrow$} & $\begin{array}{l}\text { North } \\
\text { America }\end{array}$ & $\begin{array}{l}\text { [88] Over a shorter period ( } 5 \text { years), culling } \\
\text { was a more effective strategy. Over a longer } \\
\text { period ( } 20 \text { years), both methods had similar } \\
\text { effectiveness. }\end{array}$ \\
\hline & & & & India & $\begin{array}{l}\text { [85] Fertility control was more effective than } \\
\text { culling, fertility control reduced population } \\
\text { size by over } 75 \% \text {, compared to } \sim 13 \% \text { with } \\
\text { culling over } 20 \text { years. }\end{array}$ \\
\hline & $\begin{array}{l}\text { Dog rabies } \\
\text { prevalence }\end{array}$ & $\downarrow$ & $\downarrow$ & $\begin{array}{l}\text { Multiple } \\
\text { countries }\end{array}$ & $\begin{array}{l}\text { [100] Culling was as effective as fertility control } \\
\text { combined with rabies vaccination. }\end{array}$ \\
\hline \multirow{4}{*}{$\begin{array}{l}\text { Fertility control } \\
\text { and sheltering }\end{array}$} & & Fertility control & Sheltering & & \\
\hline & \multirow{3}{*}{$\begin{array}{l}\text { Dog population } \\
\text { size }\end{array}$} & \multirow[t]{2}{*}{$\downarrow$} & \multirow[t]{2}{*}{$\downarrow$} & $\begin{array}{l}\text { Multiple } \\
\text { countries }\end{array}$ & $\begin{array}{l}\text { [96] Fertility control and adoption, through } \\
\text { sheltering, had synergistic effects. Adoption, } \\
\text { through sheltering, was the most effective } \\
\text { method when comparing the two. }\end{array}$ \\
\hline & & & & $\begin{array}{l}\text { North } \\
\text { America }\end{array}$ & $\begin{array}{l}\text { [87] Fertility control was the most effective, } \\
\text { although adoption, through sheltering, worked } \\
\text { well in combination with fertility control. }\end{array}$ \\
\hline & & $\downarrow$ & $\downarrow$ & Italy & $\begin{array}{l}\text { [90] Fertility control was the most effective, } \\
\text { reducing dog population size by } 34 \% \text {, } \\
\text { compared to only } 3 \% \text { in sheltering. }\end{array}$ \\
\hline \multirow[b]{2}{*}{$\begin{array}{l}\text { Fertility control } \\
\text { and movement } \\
\text { restriction }\end{array}$} & & Fertility control & $\begin{array}{l}\text { Movement } \\
\text { restriction }\end{array}$ & & \\
\hline & $\begin{array}{l}\text { Dog population } \\
\text { size }\end{array}$ & $\downarrow$ & $\downarrow$ & Mexico & $\begin{array}{l}\text { [89] Varying size of effect relating to neutering } \\
\text { coverage, age of dog neutering and } \\
\text { confinement level. Fertility control of owned } \\
\text { dogs and dog movement restriction were most } \\
\text { effective when used together. }\end{array}$ \\
\hline \multirow{2}{*}{$\begin{array}{l}\text { Different } \\
\text { taxation } \\
\text { methods }\end{array}$} & & $\begin{array}{l}\text { Taxation of dog } \\
\text { purchases }\end{array}$ & $\begin{array}{l}\text { Subsidy of } \\
\text { dog adoption }\end{array}$ & & \\
\hline & $\begin{array}{l}\text { Dog population } \\
\text { size }\end{array}$ & $\downarrow$ & $\downarrow$ & $\begin{array}{l}\text { No specific } \\
\text { country }\end{array}$ & $\begin{array}{l}\text { [92] Taxation of dog buyers is the most effective } \\
\text { option at reducing the number of free-roaming } \\
\text { dogs. }\end{array}$ \\
\hline
\end{tabular}

\subsubsection{Dog Population Demographics}

The effects of culling, fertility control, sheltering, taxation, and combined fertility control and movement restriction on dog population demographics were investigated through modelling studies. All used dog population size as an indicator of effect. Three modelling studies investigated the effect of culling on dog population demographics. All reported that culling decreased dog population size at the intensity modelled (see Table 4 for management coverage and length). Yoak et al.'s (2016) [85] agent-based model simulated that culling would decrease population size by $13 \%$ over 20 years [85] at current capture rates, although the intensity required to achieve this reduction is not reported. All papers reported that fertility control reduced population size at the intensity modelled. The effect varied from a minimum decrease in population size of $14 \%$ over 20 years to $78 \%$ over 20 years, depending on the neutering coverage [89]. Sheltering at the modelled intensity had little or no effect on dog population size (population decrease of $3 \%$ in 10 years [90]), or no effect $[87,90]$ ). One paper in the final corpus [92] reported that taxation of dog buyers at various intensities decreased the free-roaming dog population size. Three papers $[87,89,96]$ explored the effect of combined movement restriction 
and sheltering at various modelled intensities, all reported synergistic effects but this varied from a $5 \%$ population decrease in 30 years [96] to a $73 \%$ decrease in 20 years [89].

When sheltering was directly compared to fertility control, fertility control was more effective at reducing population size [87,90]. For example, Hogasen et al. (2013) [90] modelled that an increase in fertility control by $20-40 \%$ per year reduced the free-roaming dog population size by $34 \%$, compared to only a $3 \%$ reduction where sheltering was increased by $10 \%$ each year. In studies that directly compared the effects of culling to fertility control on dog population size, culling was less effective at reducing the population size. Yoak et al. (2016) [85] reported that fertility control decreased population size by $75 \%$, compared to approximately only $13 \%$ with culling when using model simulations with the same capture probability and intensity of intervention.

\subsubsection{Public Health Risk}

The effects of culling and fertility control on public health risk were investigated in modelling studies. All papers reported that, at various modelled intensities, culling decreased dog rabies prevalence (decreasing trend [100-102]) and rabies basic reproductive number (R0) (decreasing trend $[91,107,108])$. Fertility control at the modelled intensities also decreased public health risk. Fitzpatrick et al. (2016) [98] reported a reduction in the number of human rabies cases, estimating a 92\% decrease in five years of model simulation when an intervention coverage between $25 \%$ and $50 \%$ was modelled. Carroll et al. (2010) [100] reported that fertility control decreased the prevalence of dog rabies. The modelled intensity of fertility control required to eradicate rabies varied from maintaining $100 \%$ coverage for one month to maintaining $25 \%$ coverage for over two years. Carroll et al. (2010) [100] directly compared culling to fertility control and reported culling to be just as effective at reducing dog rabies prevalence at the modelled intensities. However, when combined with rabies vaccination, fertility control was more effective than culling at eradicating dog rabies [100].

\subsubsection{Wildlife}

One modelling study investigated the effect of fertility control on disease risk to wild animal populations. Using an agent-based model, Belsare et al. (2015) [109] reported that fertility control (at unspecified intensities) reduced the risk to the Indian fox (Vulpes bengalensis) population, using the number of canine distemper spill over events as an indicator.

\section{Discussion}

\subsection{Limitations in Assessing Dog Population Management}

This systematic review synthesises research papers investigating different dog population management methods. We determined: (1) where and when the impact of dog population management has been described in the published literature; (2) what methods were assessed and at what intensity (coverage and length of management); and (3) what effects were reported. Furthermore, we evaluated the reporting quality of the studies. Papers in the final corpus suggest that most dog population management methods were associated with some effect on the impact of interest, and mostly in a favourable direction (such as decreasing public health risk or dog population size). The interpretation of these results and assessment of the effectiveness of dog population management methods is limited due to the following reasons:

1. Few studies used a study design that would allow causation to be determined (such as intervention or certain observational studies), and many lacked an appropriate number of treatment and control groups (Table S5) and replication (Table 3). This therefore makes it challenging to distinguish between changes to a population that are caused by the management method, to incidental changes caused by other factors (e.g., reduction in population numbers over a few years caused by environmental or human related factors in the study area). 
2. Multiple indicators are used to assess the impact of dog population management (Table 1). It is therefore difficult to compare the effect of the same population management method across different studies, and even more challenging to compare different methods across studies. This makes it difficult to carry out a formal synthesis of results, such as a meta-analysis, to report the combined evidence. For example, we found that different papers reporting on the evaluation of different management methods did not use the same measurement of dog health and welfare. In this example, it does not make substantive sense to compare whether an increase in normal body condition scores of $13 \%$ (with fertility control) indicates a greater impact on dog health and welfare compared to a decrease in leukocyte counts by $4(\times 109$ cell/L) (when fertility control and sheltering are combined). This therefore makes it difficult to directly compare effects between methods.

3. Studies often investigated combinations of population management methods, such as fertility control and sheltering, and fertility control and culling. It is difficult to assess the impact of dog population management when methods are not used in isolation. Even where studies investigated one method alone, it is unclear whether other methods of dog population management were in place, such as sheltering or taxation. Culling might also be under-represented, as the method is often not reported due to lack of public acceptance (e.g., ad-hoc poisoning and drowning).

4. To effectively review the results of dog population management intervention, it is important to not only consider what method was applied, but also how the method was implemented. This means in practice that information about the intensity of management and associated costs (logistics, training, and facilities) are required in order to fully appreciate and contextualise the results. Any management method has the potential to be effective if the intensity is large enough. For example, moving $100 \%$ of the dog population into shelters every week would be much more effective than to only $15 \%$ of the population once a year. It is therefore important to consider: (i) management coverage; (ii) length of management; and (iii) cost of management when assessing the effectiveness of different methods. Many papers in the final corpus did not provided information about the coverage of management and some did not report the length of management (Table 2). Information about the cost of management was rarely provided, apart from where included as a parameter in modelling studies.

\subsection{Investigated Methods and Reported Effects of Dog Population Management}

The results of this systematic review highlight the scale and increasing interest in dog population management, which has been studied globally with an increase in the rate of publications in the last decade (Figure S1). In particular, fertility control was often investigated, this aligns with increasing interest over recent years in the use of fertility control to manage animal populations in general [110]. Although interpretation of results from the final corpus is limited, we can still draw some tentative conclusions about the impact of the different management methods.

Overall, papers reported that fertility control had positive effects on dog health and welfare, including improved body condition score and reduced presence of injuries and some pathogens. However, this method increased skin conditions and prevalence of ectoparasites. The positive effects on body condition and presence of injuries could be explained by the lack of sex hormones caused by fertility control. This results in a reduced desire to seek out mates, as well as reduced sexual competition, which can cause weight gain [111,112] and decrease aggression between individuals, respectively [113]. Additionally, as fertility control methods (such as CNR) are often combined with vaccination and antiparasitic treatment, an improved health condition may be reflected in an improvement in body condition [50]. The negative effects of fertility control on the prevalence of skin conditions could relate to the specific protocols carried out by the different population management programs, such as the conditions the dogs are kept in pre- and post-surgery and the medical treatment provided (such as antiparasitics). It is therefore important that future groups carrying out dog population management through fertility control ensure they take measures to reduce pathogen transmission in clinical facilities. 
The impact of different management methods on dog population demographics was measured solely through dog population size, allowing some level of comparison between papers. The comparison is still limited, however, as these effects were measured across different time scales, applied at different rates (e.g., neutering coverages), and to different populations of dogs (e.g., free-roaming owned and unowned or free-roaming unowned). For example, in the observational studies, the impact of fertility control varied from decreases in population size of $12 \%$ in 1.5 years to decreases in size of $40 \%$ over 12 years. Although all methods decreased population size, fertility control had the greatest effect in both observational studies $[68,69,83,84]$ and modelling studies [69,85-90]. Fertility control decreases dog population size by preventing births, therefore allowing a reduction of numbers as natural deaths occur. This is in contrast with culling and sheltering, which reduce the population size through the removal of individuals, either through death or the moving of dogs into a shelter population. When fertility control was combined with other methods, such as movement restriction and sheltering, synergistic effects were reported [89]. By increasing the rate of fertility control and restriction status of dogs, this would both reduce the opportunities for reproduction and therefore potentially reduce the birth rate even greater than if fertility control had been used alone. Culling, by increasing the death rate of a population, may cause a rapid reduction in population numbers $[85,88]$. The culling method has been criticised as ineffective at reducing populations over longer periods of time [88]. This was supported by modelling studies that directly compared fertility control and culling. These papers found that, although culling resulted in an initial decrease in dog population size (e.g., a five-year period [88]), fertility control was more effective at reducing the population size over longer periods of time (e.g., a 20-year period) $[85,88]$. This may be because individuals removed through culling are replaced by new individuals through compensatory breeding or migration from other locations [114], therefore rendering the method less effective in the longer term. It is also important to note that there were no empirical studies investigating the impact of culling on population size, all were modelling studies, and therefore have limitations in the inferences that can be made to real dog populations.

Multiple different measures were reported to assess effects on public health risk, again making it difficult to compare methods directly. Culling had decreasing effects on the various indicators of public health risk in both observational $[105,106]$ and modelling studies $[91,100-102,107,108]$. This contradicts previous literature suggesting that culling is ineffective at controlling disease in free-roaming dogs $[115,116]$. For the measurement "prevalence of visceral leishmaniasis", culling only decreased prevalence over shorter study periods of up to two years (e.g., up to $69 \%$ over 14 months [105]), and had no effect over longer periods [106]. This is potentially due to other mammalian disease reservoirs that would allow continued transmission of Leishmania infantum to the remaining dog population [106] or by the number of free-roaming dogs recovering after culling, through immigration or compensatory breeding mechanisms [114]. In addition, culling decreased both dog rabies prevalence $[100,101]$ and the basic reproductive number of rabies $[91,107,108]$ in modelling studies. However, when disease control through vaccination was included in the analysis, all papers in the final corpus reported that culling was not as effective as vaccination alone $[91,102,107,108]$ or combined vaccination and fertility control [100]. The prevalence of Echinococcus granulosus in humans, livestock, and dogs decreased where culling was combined with fertility control $[103,104]$. The two studies reported either large decline [104] in dog prevalence or complete eradication [103]. However, neither study uses experimental design or statistical analysis that would allow inference to the association between the management methods and the effect. All papers in the final corpus agreed that fertility control decreased the public health risk indicators $[99,100,117]$. The reduction in human bite cases can be linked to a reduction in sex hormones, which can in turn reduce the occurrence of aggression and dog bites [118]. The impact of sterilisation on owned dog aggression has long been debated within the literature, some studies finding a reduction in aggressive behaviour and others finding no effect, or increased aggression (see McKenzie (2010) [119] for review). In terms of free-roaming dogs, Garde et al.'s (2016) [120] behavioural observations in free-roaming dogs found no decrease in aggressive behaviour towards conspecifics or humans. The reduction in human bite cases reported in our findings may instead be due to an overall 
reduction in the free-roaming dog population size or a reduction in the number of puppies, therefore reducing protective behaviour of adult dogs [99].

\subsection{Study Quality and Recommendations for Future Work}

Good quality reporting of research methods and results is vitally important in understanding the validity and reliability of research findings. Additionally, research conclusions should be supported by appropriate study design and statistical modelling approaches. To improve reporting quality and study design, we suggest the following simple refinements for studies investigating the effectiveness of these dog population management approaches:

\subsubsection{Increase Reporting Quality}

Reporting guidelines are available for a number of biological areas (see [78,79,121-123]). In general, recommendations include reporting specific details about the experimental design, study subjects, statistical analyses, and modelling approaches. In particular, to increase reporting quality in studies investigating the impact of dog population management, we recommend reporting the following:

Power and Sample Size Calculations

We found the reporting of power calculations (11\%) and sample size calculations (11\%) to be low across the published papers. By not reporting this information, the value of the findings and recommendations resulting from the research are limited [79]. Therefore, power and sample size calculations should be clearly reported to increase reporting quality, replicability, and confidence in results.

\section{Defined Target Dog Population under Investigation Using Clear Common Terminology}

We suggest grouping dogs into: (i) unowned, free-roaming; (ii) owned, free-roaming; (iii) unowned, restricted; and (iv) owned restricted. Reporting which target dog population is under investigation would allow the effects of dog population management to be compared between different studies and between studies in different countries. This is particularly important where the definition of dog ownership might differ, for example in areas where there are community dogs that are loosely owned.

\section{Management Intensity and Cost}

Papers in the final corpus often did not explicitly state the length, coverage, and cost of the applied management method. As described above, to assess management effectiveness, it is important to report the length and coverage of management (e.g., the number of dogs neutered as a percentage of the total population). Reporting management coverage requires knowledge of the dog population size and this can be achieved through methods such as mark-recapture (see [124] for review of dog population estimation methods). Conducting population estimation requires time, manpower, and expertise in study design and analysis. Dog population management is often carried out by charities and government agencies (Table S2) and these organisations may lack the financial and logistical power, as well as the expertise to conduct such ecological methods. This might partly explain the lack of reporting of management coverage. It is also important to consider and report the population management history in the study area, as previous management may impact the effectiveness of successive management. This information may also be difficult to access, but should be reported if available.

\subsubsection{Improve Experimental and Statistical Modelling Approaches}

\section{Experimental Approaches}

Where possible, researchers should consider their experimental approach and use an intervention (e.g., randomised control trials) or observational (e.g., cohort studies) study design that allows cause 
and effect to be determined, therefore allowing assessment of the true impact of dog population management. Where appropriate for the study design (i.e., intervention and analytical observational studies), appropriate numbers of groups and replication should be included, such as multiple treatment and control groups. This ensures that any effects reported are caused by the dog population management method and not by other causes (e.g., differences in population numbers between years due to differing mortality rates because of weather or other events).

\section{Statistical Modelling Approaches}

Due to practical, logistical, and financial constraints, studies that are observational (including cohort, cross-sectional, and case-control studies) are often the only feasible options for assessing dog population management. These studies may result in datasets that include large variability due to biological processes, sampling methods, and context (e.g., the specific country the study was conducted). Statistical modelling approaches can be used to deal with the limitations in inferring causal relationships using observational data. These include controlling for variables in statistical models, or matching of additional variables. We recommend using approaches such as directed acyclic graphs $[125,126]$ to help to identify when controlling for variables in the statistical analysis is appropriate, and what variables to control for (see [127] for primer on creating acyclic graphs, dealing with measurement error, and statistically controlling for variables). For example, in a study investigating the impact of population management on dog health, it would be appropriate to control for age of dogs, as young dogs have a different probability of developing certain health conditions than older dogs. Therefore, if age were not controlled for in these analyses, the causal relationship between population management and dog health might not be observed. Additionally, process based modelling approaches have been developed to incorporate the underlying processes in the statistical analysis [128]. These modelling approaches incorporate both the sampling and biological processes that create the patterns observed in the data (e.g., hidden process models [129]), leading to better interpretation of complex causal relationships, where context creates differing outcomes. An example of datasets where hidden process models could be used includes data collected about dog population size through mark-recapture methods or citizen science [129-131]. This would therefore incorporate the processes involved in observing dogs (sampling process-e.g., detection probability) and the biological processes involved in the dogs being in the sampling area (biological process-e.g., the probability of migration, birth, or death). It is worth noting that these approaches require statistical and modelling knowledge that may be challenging for the organisations involved in dog population management to acquire/access.

\section{Conclusions}

Our systematic review found that dog population management is conducted in many countries globally [15,16], carried out by different groups (e.g., researchers and animal welfare or government agencies), applying different methods to different populations types (restricted and unrestricted) and using different indicators to monitor the impact of the intervention. It is therefore difficult to synthesise the evidence base and assess the true impact of dog population management techniques [3,16], despite the quantity of work being conducted. Very few of the reviewed studies allowed robust conclusions to be drawn. We recommend that future studies: (i) increase reporting quality; (ii) clearly define target populations; and (iii) increase the use of study design and modelling approaches that allow causality to be determined, in order that cross-study data synthesis and learning can be conducted for a stronger evidence base to support interventions.

Supplementary Materials: The following are available online at http://www.mdpi.com/2076-2615/9/12/1020/s1, Figure S1: Cumulative number of publications in the final corpus per year between 1977 and 2018, Table S1: Key words and combinations, Table S2: Papers in the final corpus by subject, dog population management method, country, economic status of that country, and funding organisation. Table S3: Number (and ratio) of published articles from the final corpus that measure the impact of the management method(s) studied. Table S4: Number 
of papers investigating combinations of dog populations. Table S5: Summary of the reporting of study quality indicators in final corpus (excluding modelling studies).

Author Contributions: Conceptualisation, L.M.S., L.M.C., and R.J.Q.; methodology, L.M.S., L.M.C., and R.J.Q.; formal analysis, L.M.S.; investigation, L.M.S.; data curation, L.M.S.; writing-original draft preparation, L.M.S.; writing-review and editing, L.M.S., L.M.C., S.H., A.M.M., P.D.V., and R.J.Q.; visualisation, L.M.S.; supervision, L.M.C. and R.J.Q.; and funding acquisition, L.M.C.

Funding: This research was funded by VIER PFOTEN International.

Acknowledgments: The authors would like to thank Steven Sait, Helen Gray, and Mary Friel for their useful comments on an earlier draft of this manuscript. We would also like to thank the three anonymous reviewers for providing valuable feedback on the manuscript.

Conflicts of Interest: The authors declare no conflict of interest. The funders were involved in the reviewing of the manuscript.

\section{References}

1. Hughes, J.; Macdonald, D.W. A review of the interactions between free-roaming domestic dogs and wildlife. Biol. Conserv. 2013, 157, 341-351. [CrossRef]

2. Matter, H.; Daniels, T. Dog ecology and population biology. In Dogs, Zoonoses and Public Health; Macpherson, C.N.L., Meslin, F.X., Wandeler, A.I., Eds.; CABI Publishing: New York, NY, USA, 2000; pp. 17-62.

3. International Companion Animal Management Coalition (ICAM). Humane Dog Population Management Guidance; ICAM: UK, 2008.

4. Belsare, A.; Gompper, M. Assessing demographic and epidemiologic parameters of rural dog populations in India during mass vaccination campaigns. Prev. Vet. Med. 2013, 111, 139-146. [CrossRef] [PubMed]

5. Jimenez, S.; Perez, A.; Gil, H.; Schantz, P.M.; Ramalle, E.; Juste, R.A. Progress in control of cystic echinococcosis in La Rioja, Spain: Decline in infection prevalences in human and animal hosts and economic costs and benefits. ACTA Trop. 2002, 83, 213-221. [CrossRef]

6. Economides, P.; Christofi, G. Experience gained and evaluation of the Echinococcosis/Hydatidosis eradication programmes in Cyprus 1971-1999. In Proceedings of the Cestode Zoonoses: Echinococcosis and Cysticercosis: An Ermergent and Global Problem; Craig, P., Pawlowski, Z., Eds.; IOS Press: Amsterdam, The Netherlands, 2002; Volume 341, pp. 367-379.

7. Morters, M.K.; McKinley, T.J.; Restif, O.; Conlan, A.J.K.; Cleaveland, S.; Hampson, K.; Whay, H.R.; Damriyasa, I.M.; Wood, J.L.N. The demography of free-roaming dog populations and applications to disease and population control. J. Appl. Ecol. 2014, 51, 1096-1106. [CrossRef] [PubMed]

8. Haydon, D.T.; Laurenson, M.K.; Sillero-Zubiri, C. Integrating epidemiology into population viability analysis: Managing the risk posed by rabies and canine distemper to the Ethiopian wolf. Conserv. Biol. 2002, 16, 1372-1385. [CrossRef]

9. Newsome, T.M.; Stephens, D.; Ballard, G.-A.; Dickman, C.R.; Fleming, P.J.S. Genetic profile of dingoes (Canis lupus dingo) and free-roaming domestic dogs (C. 1. familiaris) in the Tanami Desert, Australia. Wildl. Res. 2013, 40, 196-206. [CrossRef]

10. Doherty, T.S.; Dickman, C.R.; Glen, A.S.; Newsome, T.M.; Nimmo, D.G.; Ritchie, E.G.; Vanak, A.T.; Wirsing, A.J. The global impacts of domestic dogs on threatened vertebrates. Biol. Conserv. 2017, 210, 56-59. [CrossRef]

11. Fielding, W.; Mather, J. Stray dogs in an island community: A case study from New Providence, The Bahamas. J. Appl. Anim. Welf. Sci. 2000, 3, 305-319. [CrossRef]

12. Rodriguez-Vivas, R.I.; Ortega-Pacheco, A.; Rosado-Aguilar, J.A.; Bolio, G.M.E. Factors affecting the prevalence of mange-mite infestations in stray dogs of Yucatán, Mexico. Parasitology 2003, 115, 61-65. [CrossRef]

13. HIS. Case Study of An Incentive Program to Encourage the Sterilization of Dogs (And Cats) and Greater Attention to Animal Welfare on Abaco Island in the Bahamas; Humane Society International: Washington, DC, USA, 2001.

14. Tasker, L. Stray Animal Control Practices (Europe); WSPA and RSPCA: London, UK, 2007.

15. Dalla Villa, P.; Kahn, S.; Stuardo, L.; Iannetti, L.; Di Nardo, A.; Serpell, J.A. Free-roaming dog control among OIE-member countries. Prev. Vet. Med. 2010, 97, 58-63. [CrossRef]

16. Hiby, E.; Atema, K.N.; Brimley, R.; Hammond-Seaman, A.; Jones, M.; Rowan, A.; Fogelberg, E.; Kennedy, M.; Balaram, D.; Nel, L.; et al. Scoping review of indicators and methods of measurement used to evaluate the impact of dog population management interventions. BMC Vet. Res. 2017, 13, 1-20. [CrossRef] [PubMed] 
17. Pastoret, P.; Van Gucht, S.; Brochier, B. Eradicating rabies at source. Rev. Sci. Tech. Int. DES Epizoot. 2014, 33, 509-519.

18. Zhang, W.; Zhang, Z.; Wu, W.; Shi, B.; Li, J.; Zhou, X.; Wen, H.; McManus, D.P. Epidemiology and control of echinococcosis in central Asia, with particular reference to the People's Republic of China. ACTA Trop. 2015, 141, 235-243. [CrossRef]

19. Harhay, M.; Olliaro, P.; Costa, D.; Costa, C. Urban parasitology: Visceral leishmaniasis in Brazil. Trends Parasitol. 2011, 27, 403-409. [CrossRef]

20. Ponce-Macotela, M.; Peralta-Abarca, G.E.; Martinez-Gordillo, M.N. Giardia intestinalis and other zoonotic parasites: Prevalence in adult dogs from the southern part of Mexico City. Vet. Parasitol. 2005, 131, 1-4. [CrossRef]

21. Macpherson, C. The epidemiology and public health importance of toxocariasis: A zoonosis of global importance. Int. J. Parasitol. 2013, 43, 999-1008. [CrossRef]

22. Clarke, N.M.; Fraser, D. Animal control measures and their relationship to the reported incidence of dog bites in urban Canadian municipalities. Can. Vet. J. 2013, 54, 145-149.

23. Morales, C.; Férnandez, C.; Hernández, H.; Falcón, N. Dog bite accidents in a children hospital at Lima, Peru. Retrospective study from 1995-2009. Rev. Peru. Med. Exp. Salud Publica 2011, 28, 639-642. [CrossRef]

24. Akpinar, O.; Kapci, M.; Duman, A.; Turkdogan, K.A. Evaluation of suspected rabies animal bites and comparison of vaccination protocols. Acta Med. Mediterr. 2015, 31, 919-924.

25. Jackman, J.; Rowan, A. Free-Roaming Dogs in Developing Countries: The Benefits of Capture, Neuter, and Return Programs; Salem, D.J., Rowan, A.N., Eds.; Humane Society Press: Washington, DC, USA, 2007.

26. Garde, E.; Acosta-Jamett, G. BM Bronsvoort Review of the risks of some canine zoonoses from free-roaming dogs in the post-disaster setting of Latin America. Animals 2013, 3, 855-865. [CrossRef]

27. Gompper, M.E. The Dog-Human-Wildlife Interface: Assessing the Scope of the Problem, Oxford University Press: Oxford, UK, 2014.

28. Knobel, D.D.L.; Cleaveland, S.; Coleman, P.G.P.; Fèvre, E.M.; Meltzer, M.I.; Miranda, M.E.G.; Shaw, A.; Zinsstag, J.; Meslin, F.-X. Re-evaluating the burden of rabies in Africa and Asia. Bull. World Health Organ. 2005, 83, 360-368. [PubMed]

29. Hampson, K.; Coudeville, L.; Lembo, T.; Sambo, M.; Kieffer, A.; Attlan, M.; Barrat, J.; Blanton, J.D.; Briggs, D.J.; Cleaveland, S. Estimating the global burden of endemic canine rabies. PLoS Negl. Trop. Dis. 2015, 9, 1-20.

30. Quinnell, R.; Parasitology, O.C. Transmission, reservoir hosts and control of zoonotic visceral leishmaniasis. Parasitology 2009, 135, 1915-1934. [CrossRef] [PubMed]

31. Yang, Y.R.Y.; McManus, D.D.P.; Huang, Y.; Heath, D.D. Echinococcus granulosus Infection and Options for Control of Cystic Echinococcosis in Tibetan Communities of Western Sichuan Province, China. PLoS Negl. Trop. Dis. 2009, 3, 1-7. [CrossRef]

32. Overgaauw, P.; Nederland, V. Aspects of Toxocara epidemiology: Toxocarosis in dogs and cats. Crit. Rev. Microbiol. 1997, 23, 233-251. [CrossRef]

33. Vial, F.; Cleaveland, S.; Rasmussen, G.; Haydon, D.T. Development of vaccination strategies for the management of rabies in African wild dogs. Biol. Conserv. 2006, 131, 180-192. [CrossRef]

34. Cleaveland, S.; Appel, M.G.; Chalmers, W.S.; Chillingworth, C.; Kaare, M.; Dye, C. Serological and demographic evidence for domestic dogs as a source of canine distemper virus infection for Serengeti wildlife. Vet. Microbiol. 2000, 72, 217-227. [CrossRef]

35. Ritchie, E.; Dickman, C.; Letnic, M.; Vanak, A.T. Dogs as predators and trophic regulators. In Free-Ranging Dogs and Wildlife Conservation; Gompper, M.E., Ed.; Oxford University Press: Oxford, UK, 2014; pp. 55-68.

36. Vanak, A.; Dickman, C.; Silva-Rodriguez, E.A.; Butler, J.; Ritchie, E. Top-dogs and under-dogs: Competition between dogs and sympatric carnivores. In Free-Ranging Dogs and Wildlife Conservation; Gompper, M., Ed.; Oxford University Press: Oxford, UK, 2013; pp. 69-93.

37. Vilà, C.; Wayne, R. Hybridization between wolves and dogs. Conserv. Biol. 1999, 13, 195-198. [CrossRef]

38. Sundqvist, A.-K.; Ellegren, H.; Vila, C. Wolf or dog? Genetic identification of predators from saliva collected around bite wounds' on prey. Conserv. Genet. 2008, 9, 1275-1279. [CrossRef]

39. Sundqvist, A.; Llaneza, L.; Echegaray, J.; Beltrán, J. Hybridization between Wolves and Dogs: Impact on a Wolf Population. Ph.D. Thesis, Uppsala University, Uppsala, Sweden, 2008.

40. Vanak, A.; Gompper, M. Dogs Canis familiaris as carnivores: Their role and function in intraguild competition. Mamm. Rev. 2009, 39, 265-283. [CrossRef] 
41. Young, J.; Olson, K.; Reading, R.; Amgalanbaatar, S.; Berger, J. Is wildlife going to the dogs? Impacts of feral and free-roaming dogs on wildlife populations. Bioscience 2011, 61, 125-132. [CrossRef]

42. Manor, R.; Saltz, D. The impact of free-roaming dogs on gazelle kid/female ratio in a fragmented area. Biol. Conserv. 2004, 119, 231-236. [CrossRef]

43. Silva-Rodriguez, E.A.; Verdugo, C.; Alejandro Aleuy, O.; Sanderson, J.G.; Ortega-Solis, G.R.; Osorio-Zuniga, F.; Gonzalez-Acuna, D. Evaluating mortality sources for the Vulnerable pudu Pudu puda in Chile: Implications for the conservation of a threatened deer. ORYX 2010, 44, 97-103. [CrossRef]

44. Taborsky, M. Kiwis and dog predation: Observations in Waitangi State Forest. Notornis 1988, 35, 197-202.

45. Home, C.; Pal, R.; Sharma, R.; Suryawanshi, K.; Bhatnagar, Y.; Vanak, A. Commensal in conflict: Livestock depredation patterns by free-ranging domestic dogs in the Upper Spiti Landscape, Himachal Pradesh, India. R. Swedish Acad. Sci. 2017, 46, 655-666. [CrossRef]

46. Ciucci, P.; Boitani, L. Wolf and dog depredation on livestock in central Italy. Wildl. Soc. Bull. 1998, 26, 504-514.

47. Blair, B.; Townsend, T. Dog Predation of Domestic Sheep in Ohio. J. Range Manag. 1983, 36, 527-528. [CrossRef]

48. Bergman, D.L.; Breck, S.W.; Bender, S.C. Dogs gone wild: Feral dog damage in the United States. In Proceedings of the Thirteenth Wildlife Damage Management Conference, Saratoga Springs, NY, USA, 5 April 2009; pp. 177-183.

49. Wierzbowska, I.A.; Hedrzak, M.; Popczyk, B.; Okarma, H.; Crooks, K.R. Predation of wildlife by free-ranging domestic dogs in Polish hunting grounds and potential competition with the grey wolf. Biol. Conserv. 2016, 201, 1-9. [CrossRef]

50. Totton, S.C.; Wandeler, A.I.; Ribble, C.S.; Rosatte, R.C.; McEwen, S.A. Stray dog population health in Jodhpur, India in the wake of an animal birth control (ABC) program. Prev. Vet. Med. 2011, 98, 215-220. [CrossRef]

51. Butler, J.; Brown, W.; du Toit, J. Anthropogenic Food Subsidy to a Commensal Carnivore: The Value and Supply of Human Faeces in the Diet of Free-Ranging Dogs. Animals 2018, 8, 67. [CrossRef]

52. Rautenbach, G.H.; Boomker, J.; De Villiers, I.L. A descriptive study of the canine population in a rural town in southern africa. J. S. Afr. Vet. Assoc. 1991, 62, 158-162. [PubMed]

53. Fielding, W.; Mather, J.; Isaacs, M. Potcakes: Dog ownership in New Providence, The Bahamas; Purdue University Press: West Lafayette, IN, USA, 2005.

54. Strakova, M.; Strakova, A.; Murchison, E.P. The changing global distribution and prevalence of canine transmissible venereal tumour. BMC Vet. Res. 2014, 10, 1-10. [CrossRef] [PubMed]

55. Ortega-Pacheco, A.; Segura-Correa, J.C.; Jimenez-Coello, M.; Linde Forsberg, C. Reproductive patterns and reproductive pathologies of stray bitches in the tropics. Theriogenology 2007, 67, 382-390. [CrossRef] [PubMed]

56. Reese, J. Dogs and dog control in developing countries. In The State of the Animals; Salem, D., Rowan, A., Eds.; Humane Society Press: Washington, DC, USA, 2005; pp. 55-64.

57. Meslin, F.; Miles, M.; Vexenat, A.; Gemmell, M. Zoonoses control in dogs. In Dogs, Zoonoses and Public Health; Macpherson, C.N.L., Meslin, F.X., Wandeler, A.I., Eds.; CABI Publishing: New York, NY, USA, 2000; pp. 333-372.

58. Bögel, K.; Frucht, K.; Drysdale, G.; Remfry, J. World Health Organisation Guidelines for Dog Population Management; WHO: Geneva, Switzerland, 1990.

59. Animal Protection Act (SG No. 13/2008); Bulgarian Government: Bulgaria, 2008; pp. 1-25.

60. Italia. Legge quadro 4 agosto 1991, n. 281. Legge quadro in materia di animali d'affezione e prevenzione del randagismo. Gazzetta Ufficiale n. 203, 30 agosto 1991; Italian Government, 1991.

61. Law on Animal Welfare; Provisional Institutions of Self Government: Kosovo, 2001; pp. 1-9.

62. Peng, S.J.-L.; Lee, L.Y.-T.; Fei, A.C.-Y. Shelter Animal Management and Trends in Taiwan. J. Appl. Anim. Welf. Sci. 2012, 15, 346-357. [CrossRef]

63. Morris, K.; Wolf, J.; Gies, D. Trends in intake and outcome data for animal shelters in Colorado, 2000 to 2007. J. Am. Vet. 2011, 238, 329-336. [CrossRef]

64. Marston, L.; Bennett, P.; Coleman, G. What happens to shelter dogs? Part 2. Comparing three Melbourne welfare shelters for nonhuman animals. J. Appl. Anim. 2005, 8, 25-45. [CrossRef]

65. Barnard, S.; Pedernera, C.; Candeloro, L.; Ferri, N.; Velarde, A.; Dalla Villa, P. Development of a new welfare assessment protocol for practical application in long-term dog shelters. Vet. Rec. 2015, 178, 2-8. [CrossRef] 
66. Massei, G. Fertility control in dogs. In Zoonoses and Public Health; Macpherson, C.N.L., Meslin, F.X., Wandeler, A.I., Eds.; CABI Publishing: New York, NY, USA, 2013; pp. 259-270.

67. Barnard, S.; Chincarini, M.; Di Tommaso, L.; Di Giulio, F.; Messori, S.; Ferri, N. Free-roaming dogs control activities in one italian province (2000-2013): Is the implemented approach effective? Maced. Vet. Rev. 2015, 38, 149-158. [CrossRef]

68. Reece, J.F.; Chawla, S.K. Control of rabies in Jaipur, India, by the sterilisation and vaccination of neighbourhood dogs. Vet. Rec. 2006, 159, 379-383. [CrossRef]

69. Totton, S.C.; Wandeler, A.I.; Zinsstag, J.; Bauch, C.T.; Ribble, C.S.; Rosatte, R.C.; McEwen, S.A. Stray dog population demographics in Jodhpur, India following a population control/rabies vaccination program. Prev. Vet. Med. 2010, 97, 51-57. [CrossRef]

70. Yoak, A.J.; Reece, J.F.; Gehrt, S.D.; Hamilton, I.M. Disease control through fertility control: Secondary benefits of animal birth control in Indian street dogs. Prev. Vet. Med. 2014, 113, 152-156. [CrossRef] [PubMed]

71. Tenzin, T.; Ahmed, R.; Debnath, N.C.; Ahmed, G.; Yamage, M. Free-Roaming Dog Population Estimation and Status of the Dog Population Management and Rabies Control Program in Dhaka City, Bangladesh. PLoS Negl. Trop. Dis. 2015, 9, 1-14. [CrossRef] [PubMed]

72. Kumarapeli, V.; Awerbuch-Friedlander, T. Human rabies focusing on dog ecology-A challenge to public health in Sri Lanka. ACTA Trop. 2009, 112, 33-37. [CrossRef] [PubMed]

73. Belo, V.S.; Struchiner, C.J.; Werneck, G.L.; Teixeira Neto, R.G.; Tonelli, G.B.; de Carvalho Junior, C.G.; Nascimento Ribeiro, R.A.; da Silva, E.S. Abundance, survival, recruitment and effectiveness of sterilization of free-roaming dogs: A capture and recapture study in Brazil. PLoS ONE 2017, 12, 1-19. [CrossRef] [PubMed]

74. VIER PFOTEN International; Vienna, Austria. Personal communication, 31 April 2017.

75. Fielding, W. Knowledge of the welfare of nonhuman animals and prevalence of dog care practices in New Providence, The Bahamas. J. Appl. Anim. Welf. Sci. 2007, 10, 153-168. [CrossRef] [PubMed]

76. Slater, M.R.; Di Nardo, A.; Pediconi, O.; Villa, P.D.; Candeloro, L.; Alessandrini, B.; Del Papa, S. Free-roaming dogs and cats in central Italy: Public perceptions of the problem. Prev. Vet. Med. 2008, 84, 27-47. [CrossRef]

77. The World Bank-Countries and Economies. Available online: https://data.worldbank.org/country (accessed on 5 August 2019).

78. Moher, D.; Liberati, A.; Tetzlaff, J.; Altman, D.G. Preferred Reporting Items for Systematic Reviews and Meta-Analyses: The PRISMA Statement. PLoS Med. 2009, 151, 264-269.

79. Kilkenny, C.; Browne, W.J.; Cuthill, I.C.; Emerson, M.; Altman, D.G. Improving Bioscience Research Reporting: The ARRIVE Guidelines for Reporting Animal Research. PLoS Biol. 2010, 8, 1-5. [CrossRef]

80. Demirbas, Y.S.; Emre, B.; Kockaya, M. Integration ability of urban free-ranging dogs into adoptive families' environment. J. Vet. Behav. Appl. Res. 2014, 9, 222-227. [CrossRef]

81. Radisavljevic, K.; Vucinic, M.; Becskei, Z.; Stanojkovic, A.; Ostovic, M. Comparison of stress level indicators in blood of free-roaming dogs after transportation and housing in the new environment. J. Appl. Anim. Res. 2017, 45, 52-55. [CrossRef]

82. Airikkala-Otter, I.; Gamble, L.; Mazeri, S.; Handel, I.G.; de Bronsvoort, B.M.C.; Mellanby, R.J.; Meunier, N.V. Investigation of short-term surgical complications in a low-resource, high-volume dog sterilisation clinic in India. BMC Vet. Res. 2018, 14, 1-8. [CrossRef] [PubMed]

83. Hiby, L.R.; Reece, J.F.; Wright, R.; Jaisinghani, R.; Singh, B.; Hiby, E.F. A mark-resight survey method to estimate the roaming dog population in three cities in Rajasthan, India. BMC Vet. Res. 2011, 7, 1-9. [CrossRef] [PubMed]

84. Garcia, R.C.M.; Amaku, M.; Biondo, A.W.; Ferreira, F. Dog and cat population dynamics in an urban area: Evaluation of a birth control strategy. Pesqui. Vet. Bras. 2018, 38, 511-518. [CrossRef]

85. Yoak, A.J.; Reece, J.F.; Gehrt, S.D.; Hamilton, I.M. Optimizing free-roaming dog control programs using agent-based models. Ecol. Modell. 2016, 341, 53-61. [CrossRef]

86. Santos Baquero, O.; Marconcin, S.; Rocha, A.; de Maria Garcia, R.C. Companion animal demography and population management in Pinhais, Brazil. Prev. Vet. Med. 2018, 158, 169-177. [CrossRef]

87. Frank, J. An interactive model of human and companion animal dynamics: The ecology and economics of dog overpopulation and the human costs of addressing the problem. Hum. Ecol. 2004, 32, 107-130. [CrossRef]

88. Amaku, M.; Dias, R.A.; Ferreira, F. Dynamics and Control of Stray Dog Populations. Math. Popul. Stud. 2010, 17, 69-78. [CrossRef] 
89. Kisiel, L. Using a dog demography field study to inform the development of an agent based computer simulation. Evaluating owned dog population control interventions in a small, semi-urban community in Mexico. Master's Thesis, The University of Guelph, Guelph, ON, Canada, 2017.

90. Hogasen, H.R.; Er, C.; Di Nardo, A.; Dalla Villa, P.; Høgåsen, H.; Er, C.; Di Nardo, A.; Villa, P.D. Free-roaming dog populations: A cost-benefit model for different management options, applied to Abruzzo, Italy. Prev. Vet. Med. 2013, 112, 401-413. [CrossRef]

91. Leung, T.; Davis, S.A. Rabies Vaccination Targets for Stray Dog Populations. Front. Vet. Sci. 2017, 4, 1-10. [CrossRef]

92. Liu, S.; Chen, H. Solving Stray-Animal Problems by Economic Policies. Taipei Econ. Inq. 2016, 1-27.

93. Schurer, J.M.; Phipps, K.; Okemow, C.; Beatch, H.; Jenkins, E. Stabilizing Dog Populations and Improving Animal and Public Health Through a Participatory Approach in Indigenous Communities. Zoonoses Public Health 2015, 62, 445-455. [CrossRef]

94. Boey, J. Working with communities to improve the quality of life of British Columbia's free-roaming dogs and their people. Master's Thesis, University of Victoria, Victoria, BC, Canada, 2017.

95. Panichabhongse, P. The epidemiology of rabies in Thailand. Master's Thesis, Massey University, Palmerston North, New Zealand, 2001.

96. Santos Baquero, O.; Akamine, L.A.; Amaku, M.; Ferreira, F. Defining priorities for dog population management through mathematical modeling. Prev. Vet. Med. 2016, 123, 121-127. [CrossRef]

97. Costa, E.D.; Martins, C.M.; Cunha, G.R.; Catapan, D.C.; Ferreira, F.; Oliveira, S.T.; de Maria Garcia, R.C.; Biondo, A.W. Impact of a 3-year pet management program on pet population and owner's perception. Prev. Vet. Med. 2017, 139, 33-41. [CrossRef]

98. Fitzpatrick, M.C.; Shah, H.A.; Pandey, A.; Bilinski, A.M.; Kakkar, M.; Clark, A.D.; Townsend, J.P.; Abbas, S.S.; Galvani, A.P. One Health approach to cost-effective rabies control in India. Proc. Natl. Acad. Sci. USA 2016, 113, 14574-14581. [CrossRef] [PubMed]

99. Reece, J.F.; Chawla, S.K.; Hiby, A.R. Decline in human dog-bite cases during a street dog sterilisation programme in Jaipur, India. Vet. Rec. 2013, 172, 473-477. [CrossRef] [PubMed]

100. Carroll, M.J.; Singer, A.; Smith, G.C.; Cowan, D.P.; Massei, G. The use of immunocontraception to improve rabies eradication in urban dog populations. Wildl. Res. 2010, 37, 676-687. [CrossRef]

101. Bhunu, C.P. Impact of culling stray dogs and vaccination on the control of human rabies: A mathematical modeling approach. Int. J. Biomath. 2011, 4, 379-397. [CrossRef]

102. Zinsstag, J.; Durr, S.; Penny, M.; Mindekem, R.; Roth, F.; Gonzalez, S.; Naissengar, S.; Hattendorf, J. Transmission dynamics and economics of rabies control in dogs and humans in an African city. Proc. Natl. Acad. Sci. USA 2009, 106, 14996-15001. [CrossRef]

103. Economides, P.; Christofi, G. Evaluation of control programmes for echinococcosis/hydatidosis in Cyprus. Rev. Sci. Tech. L Off. Int. DES Epizoot. 2000, 19, 784-792. [CrossRef]

104. Polydorou, K. The anti-echinococcosis campaign in Cyprus. Trop. Anim. Health Prod. 1977, 9, 141-146. [CrossRef]

105. Melo, S.N.; Teixeira-Neto, R.G.; Werneck, G.L.; Struchiner, C.J.; Ribeiro, R.A.N.; Sousa, L.R.; de Melo, M.O.G.; Carvalho Junior, C.G.; Penaforte, K.M.; Manhani, M.N.; et al. Prevalence of visceral leishmaniasis in A population of free-roaming dogs as determined by multiple sampling efforts: A longitudinal study analyzing the effectiveness of euthanasia. Prev. Vet. Med. 2018, 161, 19-24. [CrossRef] [PubMed]

106. Ashford, D.; David, J.; Freire, M.; David, R.; Sherlock, I.; Da Conceicao, M.; Sampaio, D.; Badaro, R. Studies on control of visceral leishmaniasis: Impact of dog control on canine and human visceral leishmaniasis in Jacobina, Bahia, Brazil. Am. J. Trop. Med. Hyg. 1998, 59, 53-57. [CrossRef] [PubMed]

107. Hou, Q.; Jin, Z.; Ruan, S. Dynamics of rabies epidemics and the impact of control efforts in Guangdong Province, China. J. Theor. Biol. 2012, 300, 39-47. [CrossRef] [PubMed]

108. Zhang, J.; Jin, Z.; Sun, G.Q.; Zhou, T.; Ruan, S. Analysis of rabies in China: Transmission dynamics and control. PLoS ONE 2011, 6, 1-9. [CrossRef]

109. Belsare, A.V.; Gompper, M.E. A model-based approach for investigation and mitigation of disease spillover risks to wildlife: Dogs, foxes and canine distemper in central India. Ecol. Modell. 2015, 296, 102-112. [CrossRef]

110. Massei, G.; Cowan, D. Fertility control to mitigate human-Wildlife conflicts: A review. Wildl. Res. 2014, 41, 1-21. [CrossRef] 
111. Zoran, D. Obesity in dogs and cats: A metabolic and endocrine disorder. Vet. Clin. North Am. small Anim. 2010, 40, 221-239. [CrossRef]

112. O'farrell, V.; Peachey, E. Behavioural effects of ovariohysterectomy on bitches. J. Small Anim. Pract. 1990, 31, 595-598. [CrossRef]

113. Leney, J.; Remfry, J. (Eds.) Dog population management. In Dogs, Zoonoses and Public Health; CABI Publishing: New York, NY, USA, 2000; pp. 299-332.

114. Bögel, K.; Meslin, F. Economics of human and canine rabies elimination: Guidelines for programme orientation. Bull. World Health Organ. 1990, 68, 281-291.

115. Cleaveland, S.; Kaare, M.; Knobel, D.; Laurenson, M.K. Canine vaccination-Providing broader benefits for disease control. Vet. Microbiol. 2006, 117, 43-50. [CrossRef]

116. Hampson, K.; Dushoff, J.; Cleaveland, S.; Haydon, D.T.; Kaare, M.; Packer, C.; Dobson, A. Transmission dynamics and prospects for the elimination of canine rabies. PLoS Biol. 2009, 7, 462-471. [CrossRef] [PubMed]

117. Fitzpatrick, M.C.; Hampson, K.; Cleaveland, S.; Mzimbiri, I.; Lankester, F.; Lembo, T.; Meyers, L.A.; Paltiel, A.D.; Galvani, A.P. Cost-effectiveness of canine vaccination to prevent human rabies in rural Tanzania. Ann. Intern. Med. 2014, 160, 91-100. [CrossRef] [PubMed]

118. Goericke-Pesch, S.; Wilhelm, E.; Ludwig, C.; Desmoulins, P.; Driancourt, M.; Hoffmann, B. Evaluation of the clinical efficacy of Gonazon implants in the treatment of reproductive pathologies, behavioral problems, and suppression of reproductive function in the male dog. Theriogenology 2010, 73, 920-926. [CrossRef] [PubMed]

119. McKenzie, B. Evaluating the benefits and risks of neutering dogs and cats. CAB Rev. Perspect. Agric. Vet. Sci. Nutr. Nat. Resour. 2010, 5, 1-18. [CrossRef]

120. Garde, E.; Perez, G.E.; Vanderstichel, R.; Dalla Villa, P.F.; Serpell, J.A. Effects of surgical and chemical sterilization on the behavior of free-roaming male dogs in Puerto Natales, Chile. Prev. Vet. Med. 2016, 123, 106-120. [CrossRef]

121. Simera, I.; Moher, D.; Hoey, J.; Schulz, K.F.; Altman, D.G. A catalogue of reporting guidelines for health research. Eur. J. Clin. Investig. 2010, 40, 35-53. [CrossRef]

122. Stone, S.; Cooper, B.; Kibbler, C.; Cookson, B.; Roberts, J.; Medley, G.; Duckworth, G.; Lai, R.; Ebrahim, S.; Brown, E.; et al. The ORION statement: Guidelines for transparent reporting of outbreak reports and intervention studies of nosocomial infection. J. Antimicrob. Chemother. 2007, 59, 833-840. [CrossRef]

123. Hooijmans, C.; Leenaars, M.; Ritskes-Hoitinga, M. A gold standard publication checklist to improve the quality of animal studies, to fully integrate the three Rs, and to make systematic reviews more feasible. ALTA 2010, 38, 167-182. [CrossRef]

124. Belo, V.S.; Werneck, G.L.; da Silva, E.S.; Barbosa, D.S.; Struchiner, C.J. Population Estimation Methods for Free-Ranging Dogs: A Systematic Review. PLoS ONE 2015, 10. [CrossRef]

125. PEARL, J. Causal diagrams for empirical research. Biometrika 1995, 82, 669-688. [CrossRef]

126. Pearl, J.; Glymour, M.; Jewell, N.P. Causal Inference in Statistics: A Primer; John Wiley \& Sons: Chichester, UK, 2016; ISBN 9781119186847.

127. Rohrer, J.M. Thinking Clearly About Correlations and Causation: Graphical Causal Models for Observational Data. Adv. Methods Pract. Psychol. Sci. 2018, 1, 27-42. [CrossRef]

128. Gimenez, O.; Buckland, S.T.; Morgan, B.J.T.; Bez, N.; Bertrand, S.; Choquet, R.; Dray, S.; Etienne, M.-P.; Fewster, R.; Gosselin, F.; et al. Statistical ecology comes of age. Biol. Lett. 2014, 10, 1-4. [CrossRef]

129. Newman, K.; Buckland, S.; Lindley, S.; Thoma, L.; Fernandez, C. Hidden process models for animal population dynamics. Ecol. Appl. 2006, 16, 75-86. [CrossRef]

130. Gimenez, O.; Lebreton, J.-D.; Gaillard, J.-M.; Choquet, R.; Pradel, R. Estimating demographic parameters using hidden process dynamic models. Theor. Popul. Biol. 2012, 82, 307-316. [CrossRef]

131. Pagel, J.; Anderson, B.; O’Hara, R.; Cramer, W.; Fox, R.; Jeltsch, F.; Roy, D.; Thomas, C.; Schurr, F. Quantifying range-wide variation in population trends from local abundance surveys and widespread opportunistic occurence records. Methods Ecol. Evol. 2014, 5, 751-760. [CrossRef]

(C) 2019 by the authors. Licensee MDPI, Basel, Switzerland. This article is an open access article distributed under the terms and conditions of the Creative Commons Attribution (CC BY) license (http://creativecommons.org/licenses/by/4.0/). 